\title{
An Ultra Low Frequency Bridge for Dielectric Measurements
}

\author{
Donald J. Scheiber
}

(October 11, 1960)

\begin{abstract}
The bridge described is capable of measuring the parallel capacitance and resistance of dielectric specimens in the frequency range of 0.008 to $200 \mathrm{cps}$. It employs no earthing device and is directly connected to a three terminal oscillator. The substitution method is employed. The capabilities of the bridge are experimentally tested by measurements upon known capacitors and resistors and by comparison with results obtained using a Schering bridge near $100 \mathrm{cps}$. The apparatus is capable of accuracies previously unattained at these low frequencies. Capacitances between zero and $100 \mathrm{pf}$ may be measured to an accuracy of $\pm(0.05 \%+0.002 \mathrm{pf})$ at frequencies above $5 \mathrm{cps}$. Below $5 \mathrm{cps}$ the accuracy becomes $+\left[0.05 \%+\left(0.002+2 \times 10^{5} / f R_{D}\right) \mathrm{pf}\right]$, where $f$ is the frequency in cps and $R_{D}$ is the equivalent resistance in ohms across the detector terminals. Conductances between $10^{-9}$ and $10^{-15}$ mhos may be measured to an accuracy of about $\pm\left(1 \%+3 f \times 10^{-15}\right.$ mhos $)$ when $f \geq 0.1 \mathrm{cps}$. When $f<0.1 \mathrm{cps}$ the accuracy is about $\pm\left(1 \%+2 \times 10^{-16} \mathrm{mhos}\right)$. The dielectric constant $\left(\epsilon^{\prime}\right)$ of a specimen may be determined to an accuracy proportional to that of capacitance measurements. The loss index $\left(\epsilon^{\prime \prime}\right)$ may be determined to an accuracy of about $\pm(1 \%$ $\left.+5 \times 10^{-4} / C_{v}\right)$ when $f \geq 0.1 \mathrm{cps}$ and $\pm\left(1 \%+3 \times 10^{-5} / f C_{v}\right)$ when $f<0.1 \mathrm{cps}$. Here $C_{v}$ is the vacuum capacitance of the specimen expressed in picofarads. The effects of stray impedances shunting the bridge ratio arms, are investigated. Useful modifications of the bridge are discussed.
\end{abstract}

\section{Introduction}

Extension of the frequency range of dielectric measurements to very low frequencies is important for several reasons. For example, direct comparison can then be made between dielectric and mechanical relaxation effects in important classes of dipolar substances, such as certain polymers, for which dynamic mechanical data is often available only in this frequency region. Related to this is the study of the connection between the dielectric relaxation behavior of an amorphous material and the onset of its glass transition, the latter being closely associated with a striking slowing down of molecular motions that is reflected in a dielectric response in the ultra low frequency region. Another example is provided by the necessity of making dielectric loss measurements over the widest possible frequency range in order to determine fully the shape of dielectric loss peaks. Such studies are extremely useful in elucidating the nature of barrier systems in dipolar crystals, and in understanding the fundamental causes of dielectric loss generally. Unfortunately, little work has been carried out in the ultra low frequency range between the short time scale $d-c$ region and the ordinary $\mathrm{a}-\mathrm{c}$ bridge region (roughly 0.01 to $20 \mathrm{cps}$ ). Together with the fact that it has heretofore proven difficult to simultaneousiy measure small capacitances and conductances, this has led to a considerable gap in our knowledge of the properties of materials, and the nature of certain molecular mechanisms.

It is also clear that our knowledge of the properties of components; e.g., multimegohm resistors, is far from complete in the ultra low frequency range. The bridge discussed here is capable of providing information of this type.
A major objective of this paper is to describe an $a-c$ bridge that is capable of making accurate measurements of capacitance and (low) conductance between 0.01 and 200 cps, but which is neither cumbersome nor unnecessarily time-consuming to operate. The bridge described meets these requirements, and is therefore well suited for carrying out investigations on the dielectric properties of materials.

Some relevant background information will now be given in order to indicate the nature of earlier bridge designs, and certain problems that are encountered when dealing with ultra low frequency bridge measurements.

At frequencies below about $10 \mathrm{cps}$, transformer coupling of a bridge and a generator becomes ineffectual. At lower frequencies, the bridge and generator must be directly coupled. The first successful effort to measure dielectric properties down to $0.1 \mathrm{cps}$ was reported by Volger, Stevels, and van Amerongen [1] ${ }^{1}$ in 1953. These workers were primarily interested in measurements on high-loss inorganic glasses. They used a three terminal $R C$ oscillator directly coupled to a series resistancecapacitance bridge equipped with a Wagner earthing device. A galvanometer served as the detector. Although explicit details concerning the conductance range and sensitivity are not reported, it is apparent that this arrangement is not well suited for the measurement of low conductances, and it is desirable to extend the range to lower frequencies.

In 1955, Weingarten [2] briefly described apparatus which he had employed to measure the dielectric properties of glasses down to 0.008 cps. Heroux [3]

The figures in brackets indicate the literature references at the end of this paper. 
also gives a description of Weingarten's bridge. This three terminal bridge employed no separate guard balancing circuit, such as a Wagner earthing device. A conductance balancing system somewhat similar to that of Cole and Gross [4] was used. The low-frequency apparatus designed by Weingarten was evidently not subjected to a thorough analysis. In particular, an investigation of the effect of impedances to ground within the generator was not given. Since the three terminal generator employed is directly coupled to the bridge, these impedances shunt the bridge's ratio arms, and therefore must not be ignored. However, as will be seen below, Weingarten's basic design is a promising one. When slightly modified and subjected to careful analysis, it permits ready use of the reliable substitution method of measurement, does not require the use of a Wagner earthing device, which is exceedingly wasteful of time at low frequencies, and yields highly accurate results in the capacitance and conductance range of principal interest after certain well-defined and straightforward corrections are applied.

Nakajima and Kondo [5] circumvent the impedances-to-ground problem by operating the generator in a two terminal manner. Their bridge (briefly described elsewhere by Nakajima and Saito [6]) utilizes a Wagner earthing device, a conductance shifter, and an ungrounded or "floating" detector. Dielectric measurements with this apparatus are carried out by the direct method. The substitution method is preferable to the direct method if precise measurements of low conductance are desired. Also, it is desirable to ground one terminal of the detector. As implied earlier, the Wagner earthing device, while sound and accurate, leads to excessive measurement times in the lower end of the frequency range (see below). Hence we chose to use Weingarten's basic design, coupled with a detailed circuit analysis.

The following example indicates why a Wagner earthing device was not employed in the ultra low frequency bridge. At $0.1 \mathrm{cps}$, using the substitution method with no earthing device, about one half hour is necessary to complete the two balances that are required. With the earthing device, four balancing operations are necessary, consuming at least one hour, even if interaction between the balances is neglected. At $0.01 \mathrm{cps}$, the use of the earthing device is outright prohibitive from the standpoint of measurement time. $^{2}$

\section{Bridge Circuit and Construction}

\subsection{Bridge Circuit}

Figure 1 shows the circuit diagram of the bridge and the output circuit of the three-terminal generator. The values of the components used are given in table 1 . In figure $1, C_{x}$ and $R_{x}$ represent the

\footnotetext{
${ }^{2}$ At frequencies above about $5 \mathrm{cps}$, the objection to the earthing device mentioned
above disappears, and some benefit accrues from its use since the bridge equations are then simplified. Accordingly, a brief discussion of the application of this device then simplified. Accordingly, a brief discussion of the application of this device
to the present bridge is given in appendix 7.5 for workers interested principally in the frequency range near and above $5 \mathrm{cps}$.
}

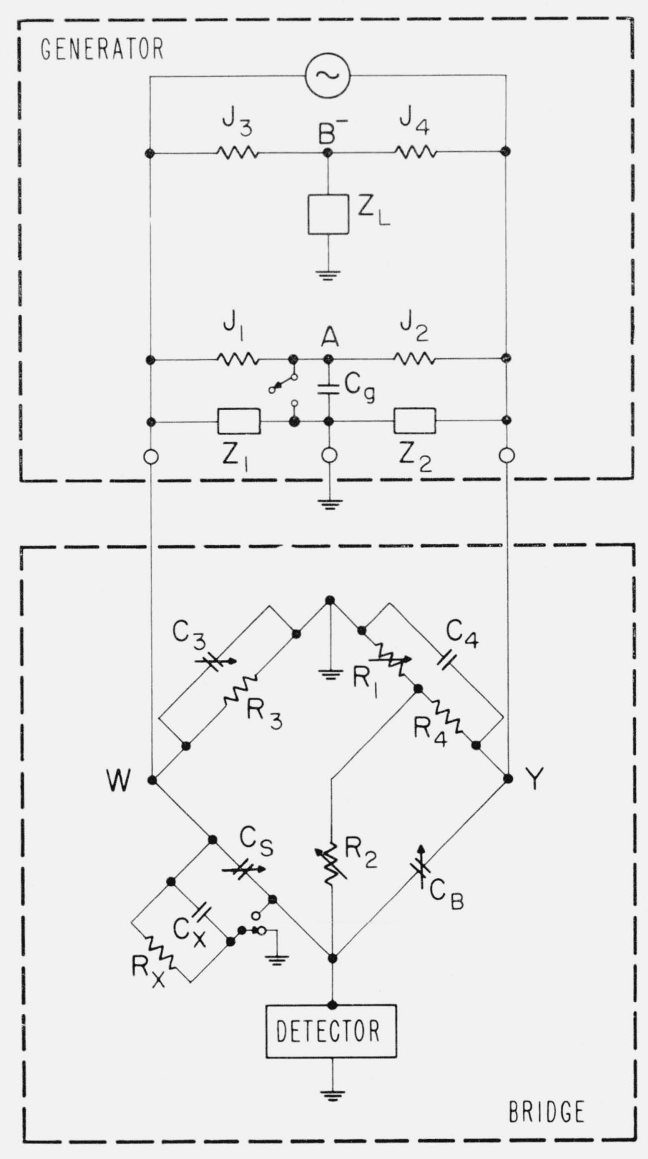

Figure 1. Circuit diagram of low frequency apparatus. Values of components are given in table 1.

parallel capacitance and resistance to be measured. $R_{3}$ and $R_{4}$ are precision wire wound, $10^{5} \mathrm{ohm}$ resistors. $C_{3}$ and $C_{4}$ include all capacitances to ground from the points $\mathrm{W}$ and $\mathrm{Y}$, respectively. $C_{3}$ also includes a two terminal variable capacitor, calibrated to \pm 0.1 pf over the range of 100 to $1000 \mathrm{pf}^{3}$ From figure 1 , it is apparent that all lead capacitances to ground within the bridge, with one exception, are either across the resistive arms and therefore included in

TABLE 1. Values of components in figure 1

\begin{tabular}{l|l}
\hline \hline & \\
$J_{1}, J_{2}$ & $15.8 \mathrm{kohm} \pm 1 \%$ \\
$J_{3}, J_{4}$ & $15 \mathrm{kohm} \pm 10^{\circ} \%$ \\
$R_{3}$ & $1.0019 \times 10^{5} \mathrm{ohms}$ \\
$R_{4}$ & $1.0012 \times 10^{5} \mathrm{ohms}$ \\
$R_{1}$ & 0 to 111.0 ohms in $0.1 \mathrm{ohm} \mathrm{steps}$ \\
$R_{2}$ & Interchangeable resistor $-10^{6}, 10^{7}, 10^{5}, 10^{9}$, \\
$C_{g}$ & $10^{10}$ ohms. \\
$C_{s}, C_{B}$ & $10 \mathrm{f} \pm 1 \%$ \\
&
\end{tabular}

${ }^{3}$ One picofarad $=$ one $p f=$ one $\mu \mu \mathrm{f}$. 
$C_{3}$ and $C_{4}$, or across the detector terminals. The exception is the capacitance to ground from the junction of $R_{1}, R_{2}$, and $R_{4}$. Such capacitance is in parallel with $R_{1}$, which has a maximum value of about 100 ohms. It can be shown that a capacitance of 1000 pf across $R_{1}$ would not detectably affect the bridge balance even at $1000 \mathrm{cps}$. The lead capacitance in question is certainly less than 1000 pf and therefore is ignored.

The bridge is balanced when the voltage between the detector terminals is zero. Capacitive balance is essentially achieved when $\left(C_{s}+C_{x}\right)$ equals $C_{B}$. The current in $R_{x}$ is balanced by inserting an equal and opposite current into the detector terminals. This is accomplished by the network consisting of $R_{1}, R_{2}$, and $R_{4}$. The gross magnitude of this current is controlled by the value of $R_{2}\left(10^{6}\right.$ to $\left.10^{10} \mathrm{ohms}\right)$. $R_{1}$ is variable in $0.1 \mathrm{ohm}$ steps from 0 to $111 \mathrm{ohms}$, and serves as a fine adjustment on the current. This arrangement provides a relatively smooth and continuous resistive balance.

The three-terminal generator employed is a Hewlett-Packard model 202A. The output circuit of the generator, as received, is included in figure 1. This circuit, especially $J_{1}, J_{2}$, and $C_{g}$, affects the operation of the bridge, and should be regarded electrically as part of the bridge. It will be shown later that it is desirable to operate the bridge with $C_{g}$ shorted. The resistive components of the generator circuit are denoted by $J$ 's rather than $R$ 's in order to emphasize the fact that they are physically part of the generator circuit, not part of the external bridge.

Null detection. Between 200 and 5 cps the detector system is a battery powered low noise preamplifier (Tektronix Inc., type 122) followed by a wave analyzer (General Radio Co., type 736-A). An unbalance voltage across the detector terminals of about a microvolt peak-to-peak can be detected. The preamplifier has an input impedance of $20 \mathrm{meg}$ paralleled by $50 \mathrm{pf}$.

Below 5 cps a d-c voltmeter is employed (Beckman Ultrohmeter). The unbalance signal appears as a periodic deflection on the indicating meter. On the most sensitive range, an unbalance signal of about 2 $\times 10^{-5}$ volts can be detected. An input resistance of $10^{11} \mathrm{ohms}$ is employed.

Below $0.1 \mathrm{cps}$ the output of the d-c voltmeter is monitored by a recording potentiometer. With the recording system employed, an unbalance signal of about $2 \times 10^{-5}$ volts peak-to-peak can be detected. Since considerable time is spent in balancing, measurements below $0.1 \mathrm{cps}$ are made only when the results are of extreme interest.

\subsection{Construction}

Care was taken to arrange the components as symmetrically and compactly as possible, and to keep capacitances to ground low. Each component is guarded from others by grounded shields ${ }^{4}$ to minimize capacitive coupling.

${ }^{4}$ The internal shields of the bridge are not shown in fig. 1 in order to simplify the drawing.
The rotors of the precision three-terminal variable capacitors $C_{s}$ and $C_{B}$ are connected together at the detector terminals. These capacitors are so connected because the capacitance to ground from the stators is more nearly independent of the setting than that from the rotors. ${ }^{5}$ Connection in this manner assures that the lead capacitances, $C_{3}$ and $C_{4}$, are nearly independent of the setting of the capacitors $C_{s}$ and $C_{B}$.

The variable resistor, $R_{1}$, is a General Radio Company compensated decade resistor, type $670-\mathrm{F}$. The "Low" terminal of the resistor is connected to ground.

The interchangeable resistors $\left(R_{2}\right)$ are mounted on a two pole, five position, selector switch and the d-c resistance at each setting is known to better than 0.1 percent. The $10^{6}$ and $10^{7} \mathrm{ohm}$ resistors are wire wound; the $10^{8}, 10^{9}$, and $10^{10}$ resistors are composition types.

The capacitors, $C_{s}$ and $C_{B}$, are calibrated against NBS reference standards to a precision of \pm 0.001 pf.

The method employed to switch the unknown "in" and "out" is such that when the unknown is "out" it is in shunt with $R_{3}$. (See fig. 1.) This is taken into account in deriving the bridge equations. It would be unwise to place a switch in the lead connecting W with the unknown, for switching at this point would alter the capacitance $C_{3}$ by an undetermined amount. The change in $C_{3}$ would be equal to the capacitance to ground of the lead and electrode connecting the sample to the switch. It would be ill-advised to change $C_{3}$ by an undetermined amount, since by analogy with a Schering bridge, this would affect the measurement of resistance. Similarly, one may discard the possibility of simply disconnecting the lead between the unknown and the detector terminal and allowing it to "float", or disconnecting both leads to the unknown. All of these possibilities lead to undefined changes in $C_{3}$ and were therefore not employed.

The resistors $J_{1}$ and $J_{2}$, which come with the generator, are "one per cent" resistors. Since under some conditions of operation these resistors are essentially the bridge's ratio arms, it is perhaps desirable to remove them from the generator and replace them with externally mounted, matched, precision resistors having magnitudes near 15.8 kohms. In this work they were not replaced since they were found to be adequately matched. Inside the generator, their values change slightly after the generator is turned on; but they remain quite stable once thermal equilibrium is established.

\section{Bridge Equations}

\subsection{Effects of the Generator Output Circuit}

The generator circuit has no connection to ground other than through the capacitor $C_{g}$, or through the stray impedances $Z_{1}, Z_{2}$, and $Z_{L}$, shown in figure 1 .

${ }^{5}$ With settings of capacitor $C_{B}$ between $20 \mathrm{pf}$ and $110 \mathrm{pf}$ the capacitance between the rotor and ground increased from 53.10 to $53.87 \mathrm{pf}$ while the capacitance between the stator and ground increased from 17.63 to $17.75 \mathrm{pf}$. 
The impedances, $Z_{1}$ and $Z_{2}$, shunt the ratio arms and may alter the impedance of the arms. The bridge equations will be worked out without taking formal cognizance of $Z_{1}$ and $Z_{2}$, but in such a way that their possible effects may be determined.

The impedance $Z_{L}$ is believed to be primarily capacitive in nature since $B^{-}$is connected to the shield of the generator's power transformer. If $Z_{L}$ be regarded as a capacitor, then we see that the network $J_{3}, Z_{L}, J_{4}$ is similar to the network $J_{1}, C_{g}$, $J_{2}$. Thus, if one establishes the effects of the network $J_{1}, C_{\mathrm{g}}, J_{2}$ upon the bridge the effect of $J_{3}, Z_{L}$, $J_{4}$ will have been at least qualitatively predicted.

Since there is no direct connection to ground in the generator, one may ground any point of the output circuit. It is obvious from figure 1 that the network $J_{1}, C_{g}, J_{2}$ will give rise to frequencydependent parallel capacitive and resistive shunts across $R_{3}$ and $\left(R_{4}+R_{1}\right)$. (This is easily demonstrated by a $\mathrm{Y} \rightarrow \Delta$ transformation.) This will clearly complicate the bridge equation and as is demonstrated later gives rise to undesirable frequency dependent "correction terms". If $C_{g}$ is removed $\left(C_{g}=0\right)$ the output of the "floating" generator is noisy and somewhat unstable, which is undesirable. These difficulties are avoided if one grounds point A of figure 1. This shorts out $C_{g}\left(C_{g}=\infty\right)$ so that $J_{1}$ and $J_{2}$ are placed across $R_{3}$ and $\left(R_{4}+R_{1}\right)$. Since $J_{1}$ and $J_{2}$ are $15.8 \mathrm{kohm}$ and $R_{3}$ and $R_{4}$ are $100 \mathrm{kohm}$, $J_{1}$ and $J_{2}$ essentially become ratio arms while $R_{3}$, $R_{1}$, and $R_{4}$ serve as part of the conductance balancing network. This is advantageous since variations in $R_{1}$ will now cause less change in the equivalent resistance of the arm. In view of these facts, in practice the bridge is operated with $C_{g}$ shorted.

Nevertheless, the bridge equations will first be worked out for the case when $C_{g}$ is in the circuit. This procedure yields general equations which (a) clearly show the effects of $C_{g}$, (b) are useful in checking out the bridge, (c) qualitatively predict the possible effects of the network $J_{3}, Z_{L}, J_{4}$, and (d) can readily be simplified for the case $C_{g}=\infty$, the mode of operation to be adopted in making routine measurements.

\subsection{Derivation of Balance Equations with $C_{g}=1 \mu f$}

The bridge is balanced by varying $C_{s}, C_{B}, R_{1}$, and $R_{2}$. The value of $C_{B}$ and $\dot{R}_{2}$ used depends upon the magnitude of $C_{x}$ and $R_{x}$. The values of $R_{2}$ and $C_{B}$ are not changed when switching the unknown in and out.

It is convenient to first transform the $\mathrm{Y}$ network formed by $R_{1}, R_{2}$, and $R_{4}$ into a $\Delta$ network as shown in figure 2(a). The equivalent resistance $R_{A}$ is in parallel with $C_{4}, R_{B}$ in parallel with $C_{B}$, and $R_{E}$ across the detector terminals. These resistances have values given by

$$
\begin{aligned}
& R_{A}=R_{1}+R_{4}+\frac{R_{1} R_{4}}{R_{2}} \\
& R_{B}=R_{4}+R_{2}+\frac{R_{4} R_{2}}{R_{1}} \\
& R_{E}=R_{2}+R_{1}+\frac{R_{2} R_{1}}{R_{4}}
\end{aligned}
$$

Lumping the impedances in each of the arms, the bridge is now equivalent to the circuit shown in figure 2(b). Here $Z_{P}$ represents the lumped impedance of the standard capacitor and the unknown when it is in parallel with $C_{s}$. The balance condition, derived in appendix 7.1, is

$$
\frac{1}{Z_{P}}\left(r+\frac{Z_{g}}{Z_{A}}(1+r)+\frac{J_{1}}{Z_{A}}\right)=\frac{1}{Z_{B}}\left(1+\frac{Z_{g}}{Z_{3}}(1+r)+\frac{J_{1}}{Z_{3}}\right),
$$

where

$$
r=J_{1} / J_{2} .
$$
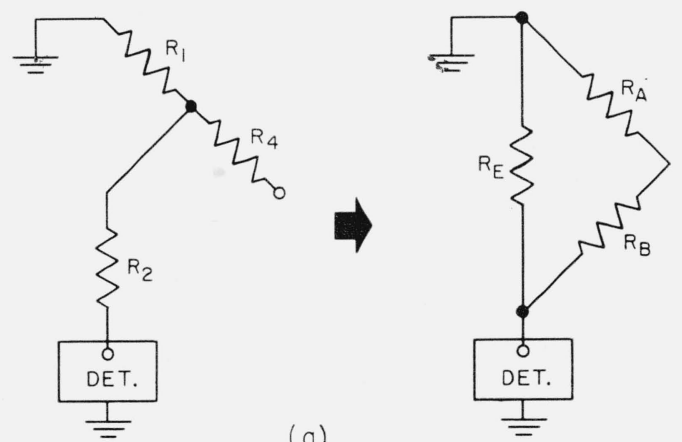

(a)

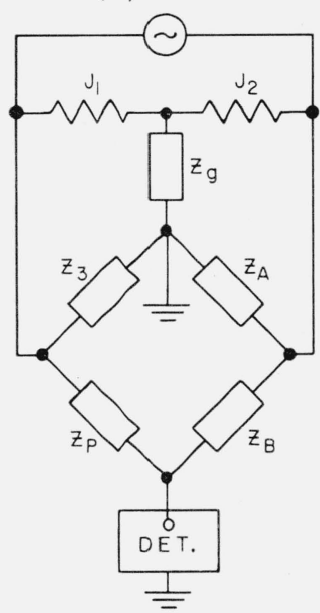

(b)

Figure 2. Network transformations.

a) Transformation of resistive Y network into a $\Delta$ network.

b) Equivalent bridge circuit after transformation. The 7's represent lumped impedances. 
The impedances ${ }^{6}$ in terms of parallel components, are given by

$$
\frac{1}{Z_{i}}=\frac{1}{R_{i}}+j \omega C_{i}
$$

where index $i$ has values $P, B$, and 3 , and

$$
\begin{aligned}
& \frac{1}{Z_{A}}=\frac{1}{R_{A}}+j \omega C_{4}, \\
& Z_{g}=-\frac{\jmath}{\omega C_{g}} .
\end{aligned}
$$

Substituting these values into eq (2) one obtains the following, upon separation of real and imaginary parts

$$
\begin{aligned}
& C_{P}\left(r+\frac{J_{1}}{R_{A}}+(1+r) \frac{C_{4}}{C_{g}}\right)+\frac{1}{R_{P}}\left(J_{1} C_{4}-\frac{(1+r)}{\omega^{2} R_{A} C_{g}}\right) \\
= & C_{B}\left(1+\frac{J_{1}}{R_{3}}+(1+r) \frac{C_{3}}{C_{g}}\right)+\frac{1}{R_{B}}\left(J_{1} C_{3}-\frac{(1+r)}{\omega^{2} R_{3} C_{g}}\right),
\end{aligned}
$$

and

$$
\begin{aligned}
& \frac{1}{R_{P}}\left(r+\frac{J_{1}}{R_{A}}+(1+r) \frac{C_{4}}{C_{g}}\right)+C_{P}\left(\frac{(1+r)}{R_{A} C_{g}}-\omega^{2} J_{1} C_{4}\right) \\
= & \frac{1}{R_{B}}\left(1+\frac{J_{1}}{R_{3}}+(1+r) \frac{C_{3}}{C_{g}}\right)+C_{B}\left(\frac{(1+r)}{R_{3} C_{g}}-\omega^{2} J_{1} C_{3}\right) .
\end{aligned}
$$

Since the substitution method is employed, equations (6) and (7) apply with the unknown across $C_{s}$ and with it disconnected from across $C_{s}$.

With the unknown 'across $C_{s}$ let the values of $R_{1}$ and $C_{s}$ lat balance be denoted as $R_{1}^{\prime \prime}$ and $C_{s}^{\prime \prime}$ respectively. The variable factors of eqs (6) and (7) become

$$
\begin{gathered}
\frac{1}{R_{P}}=\frac{1}{R_{x}}+\frac{1}{R_{s}} \\
R_{A}=R_{A}^{\prime \prime} \\
R_{B}=R_{B}^{\prime \prime} \\
C_{P}=C_{s}^{\prime \prime}+C_{x}
\end{gathered}
$$

where $R_{A}^{\prime \prime}$ and $R_{B}^{\prime \prime}$ may be computed simply from equations (1) substituting $R_{1}^{\prime \prime}$ for $R_{1}$. The parallel resistance, $R_{s}$, of the capacitor $C_{s}$ is included in the equation for $R_{P}{ }^{-1}$. Subsequently, it is assumed that $R_{s}$ is independent of the setting of $C_{s}$.

With the unknown "out" we denote the value of $R_{1}$ and $C_{s}$ at balance as $R_{1}^{\prime}$ and $C_{s}^{\prime}$ respectively.

6 The notation $j=\sqrt{-1}$ is employed. Unless otherwise stated the units of resistance $(R)$ is ohms; capacitance $(C)$ farads; and frequency $(f)$, cps.
The variable factors in equations (6) and (7) become

$$
\begin{aligned}
& R_{P}=R_{s} \\
& R_{A}=R_{A}^{\prime} \\
& R_{B}=R_{B}^{\prime} \\
& C_{P}=C_{s}^{\prime} .
\end{aligned}
$$

Since the unknown now shunts $R_{3},\left(R_{3}{ }^{-1}+R_{x}{ }^{-1}\right)$ replaces $R_{3}^{-1}$. Furthermore, $C_{x}$ now shunts $R_{3}$. The magnitude of $C_{3}$ is held constant, however, by decreasing the setting of the variable capacitor across $R_{3}$ by $C_{x}$. By obtaining a capacitive balance, $C_{s}^{\prime}$, before $C_{3}$ is adjusted, $C_{x}$ may be determined to a sufficient accuracy as $\left(C_{s}^{\prime}-C_{s}^{\prime \prime}\right) . \quad C_{3}$ is then adjusted by this amount. (This procedure is possible since the capacitance balance $C_{s}$ is practically independent of these changes in $C_{3}$.)

Substituting eqs (8) and (9) into (6) and (7) results in four equations from which exact solutions for $R_{x}$ and $C_{x}$ may be obtained. The exact solutions are given in appendix 7.2 , where it is shown that certain terms are small and may be omitted. The parallel capacitance, $C_{x}$, and conductance, $G_{x}$, of the unknown are given by

$$
\begin{aligned}
C_{x}=\Delta C_{s}+C_{s}^{\prime} \frac{\Delta R_{1}}{R_{4}} K+\left(C_{3}-C_{4}\right) \frac{\Delta R_{1}}{R_{2}} K \\
+\frac{\Delta R_{1}}{R_{2}}\left(R_{3}-r R_{4}-R_{1}^{\prime \prime}\right) L
\end{aligned}
$$

and

$$
G_{x}=\frac{1}{R_{x}}=\frac{\Delta R_{1}}{R_{2} R_{4}}\left[1+C_{s}^{\prime} R_{2} F\left(1+\frac{\omega^{2} C_{4} C_{g} J_{1}^{2}}{2}\right)\right]
$$

where

$$
\begin{gathered}
\Delta C_{s}=C_{s}^{\prime}-C_{s}^{\prime \prime} \\
\Delta R_{1}=R_{1}^{\prime \prime}-R_{1}^{\prime} \\
\omega=2 \pi f, \\
K=\frac{1+\omega^{2} \tau_{0}^{2}+R_{4} / J_{2}}{\left(1+\omega^{2} \tau_{0}^{2}\right)\left(1+R_{4} / J_{2}\right)} \\
L=\frac{C_{g}}{2 R_{4}} /\left(1+\omega^{2} \tau_{0}^{2}\right), \\
F=\frac{\omega^{2} \tau_{0}^{2}}{1+\omega^{2} \tau_{0}^{2}}\left[\frac{2}{C_{g} R_{4}\left(1+J_{1} / R_{4}\right)^{2}}\right],
\end{gathered}
$$

and

$$
\omega \tau_{0}=\frac{\omega R_{4} C_{g}}{2}\left[1+J_{1} / R_{4}\right]
$$

In eq (10) terms which contribute no more than 0.001 pf have been omitted. In eq (11) terms contributing 0.2 percent or less have been dropped. 


\subsection{Evaluation of Terms in the Balance Equations with $\mathrm{C}_{g}=1 \mu \mathrm{f}$}

Equations (10) and (11) can be placed in better perspective by numerical evaluation of the quantities $F, K$, and $L$.

Using the values of the bridge components given in table 1, eq (16) gives

$$
\omega^{2} \tau_{0}^{2}=0.132 f^{2} .
$$

Similarly, $F, K$, and $L$ become

$$
\begin{gathered}
F=15.0 \frac{\omega^{2} \tau_{0}{ }^{2}}{1+\omega^{2} \tau_{0}{ }^{2}}(\text { radians } / \mathrm{sec}), \\
K=\frac{\omega^{2} \tau_{0}{ }^{2}+7.33}{\left(1+\omega^{2} \tau_{0}^{2}\right) 7.33}, \\
L=\frac{5}{\left(1+\omega^{2} \tau_{0}^{2}\right)}(\mathrm{pf} / \mathrm{ohm}) .
\end{gathered}
$$

The function $F$ has a high frequency maximum value of about $15 \mathrm{rad} / \mathrm{sec}$ and drops sigmoidally toward zero at low frequencies. $L$ is also a sigmoidal function which is zero at low frequencies and $5 \mathrm{pf} /$ ohm at low frequencies. Both $F$ and $L$ are half their maximum values when $f=2.8 \mathrm{cps}$. The quantity $K$ is plotted against frequency in figure 3 . It has a value of 0.136 at high frequencies and rises sigmoidally toward unity at low frequencies. Also shown in figure 3 is the value of $K$ calculated for other values of $C_{g}$, along with experimental points. The significance of these points is discussed in section $4.2 \mathrm{a}$.

\section{a. Capacitance Equation}

In the equation for capacitance, eq (10),

$$
\begin{aligned}
C_{x}=\Delta C_{s}+C_{s}^{\prime} \Delta R_{1} K / R_{4}+\left(C_{3}-C_{4}\right) \Delta R_{1} K / R_{2} \\
+\left(R_{3}-R_{1}^{\prime \prime}-r R_{4}\right) \Delta R_{1} L / R_{2},
\end{aligned}
$$

it is apparent that $\Delta C_{s}$ is ordinarily the predominant term and the other three are "correction" terms. Let us maximize these correction terms in order to evaluate their importance.

The term $C_{s}^{\prime} \Delta R_{1} K / R_{4}$ has a maximum value of only $0.138 \mathrm{pf}$. This value is achieved when $\Delta R_{1}$ is $110 \mathrm{ohms}, K$ is unity, and $C_{s}^{\prime}$ is $125 \mathrm{pf}$. This is usually the most important of the correction terms.

The magnitude of the correction term, $\left(C_{3}-C_{4}\right)$ $\Delta R_{1} K / R_{2}$, depends upon the unbalance $\left(C_{3}-C_{4}\right)$, which can be experimentally determined or eliminated. The procedure is to short out $C_{g}$, and switch the unknown out;i.e., across $R_{3}$. At high frequencies (200 cps), with $C_{g}=\infty$, eq (7) to a close approximation becomes

$$
\frac{R_{1}}{R_{2} R_{4}}\left[\frac{1}{J_{1}}+\frac{1}{R_{3}}\right]=C_{s} \omega^{2}\left[C_{3}-C_{4}\right] .
$$

If one sets $R_{1}$ to zero and balances the bridge at $200 \mathrm{cps}$ by adjusting $C_{3}$ (this is the method employed

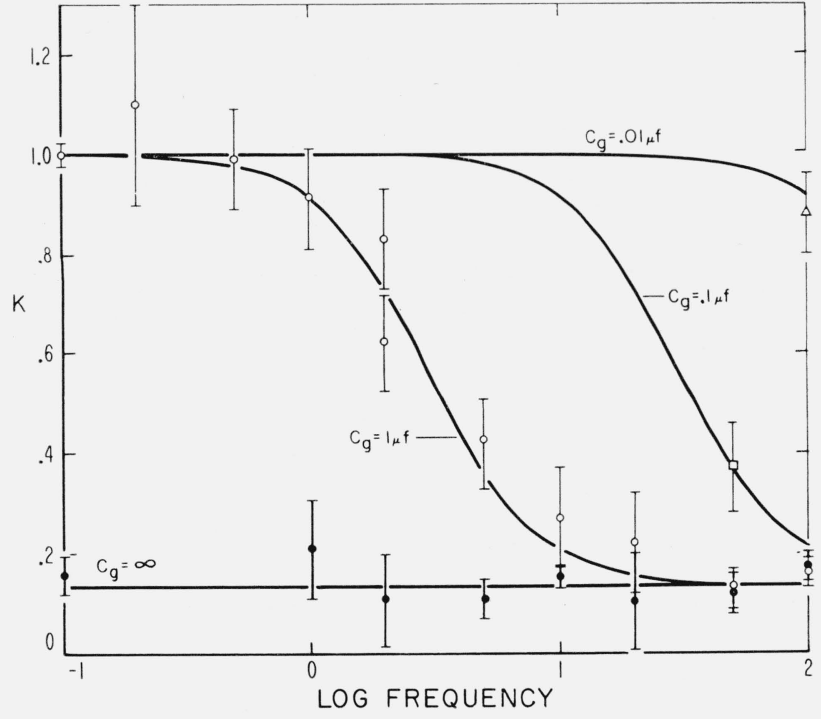

Figure 3. Frequency dependence of the largest correction term in the expression for $\mathrm{C}_{x}$.

$K$ determines the frequency dependence of the main correction term of eq (10). The solid lines are calculated using the indicated values of $C_{g}$. The experimental points, along with an indication of the experimental error, are shown.

With $C_{g}=1 \mu \mathrm{f}$, the correction term $C_{s}^{\prime} K \Delta R_{1} / R_{4}$ is strongly frequency dependent, and can attain a maximum value at low frequencies of $0.138 \mathrm{pf}(K=1)$ In practice, the bridge is operated with $C_{g}=\infty$ so that $C_{s}^{\prime} K \Delta R_{1} / R_{4}$ is independent of frequency, has a maximum value of only 0.019 pf, and can be determined to better than $0.001 \mathrm{pf}$

in balancing a Schering bridge), then $\left(C_{3}-C_{4}\right)$ becomes zero. Consequently, the correction term is zero. This desirable condition may become disturbed at lower frequencies however, since $C_{x}$, which adds to $C_{3}$, may have significant frequency dependence. Under this condition the magnitude of $\left(C_{3}-\right.$ $\left.C_{4}\right)$ becomes $\left(C_{3}-C_{4}\right)=\left[C_{x}(f)-C_{x}(200 \mathrm{cps})\right]$, where $C_{x}(f)$ is the value of $C_{x}$ at the frequency $f$. Even if $\left(C_{3}-C_{4}\right)$ were $100 \mathrm{pf}$, then, with $C_{g}=1 \mu \mathrm{f}$, the maximum value of the correction term $\left(C_{3}-C_{4}\right) K \Delta R_{1} / R_{2}$ is only $0.011 \mathrm{pf}^{7}$

The correction term, $\left(R_{3}-r R_{4}-R_{1}{ }^{\prime \prime}\right) \Delta R_{1} L / R_{2}$, depends critically upon the unbalance in the resistors $J_{1}$ and $J_{2}$, and in $R_{3}$ and $R_{4}$. The ratio $r$ is $0.99859 \pm$ .00004 (See section $4.2 \mathrm{a}$ ). Using the values of $R_{3}$ and $R_{4}$ given in table $1,\left(R_{3}-r R_{4}-R_{1}{ }^{\prime \prime}\right)$ becomes $\left(211\right.$ ohms $\left.-R_{1}^{\prime \prime}\right) \pm 10$ ohms. The maximum possible value of this correction term is therefore 0.055 pf.

Summarizing the above calculations, it is apparent that in most practical cases the correction terms of eq (10) are small. The largest of these terms, $C_{s}^{\prime} \Delta R_{1} K / R_{4}$, has a maximum value of only $0.138 \mathrm{pf}$.

\section{b. Conductance equation}

In the expression for the conductance, eq (11),

$$
G_{x}=\frac{\Delta R_{1}}{R_{2} R_{4}}\left[1+C_{s}^{\prime} R_{2} F\left(1+\frac{\omega^{2} C_{4} C_{g} J_{1}^{2}}{2}\right)\right],
$$

${ }^{7}$ Further consideration of the possible frequency dependence of $\left(C_{3}-C_{4}\right)$ due to other factors is given in section $4.2 \mathrm{~b}$. 
the product $C_{s}^{\prime} R_{2} F\left(1+\omega^{2} C_{4} C_{g} J_{1}^{2} / 2\right)$ may be regarded as a correction term. Because of sensitivity considerations, discussed in section 3.5, the $10^{9}$ and $10^{10} \mathrm{ohm}$ values of $R_{2}$ are never used above $5 \mathrm{cps}$. (Their use is also confined to low frequencies because of possible frequency dependence in their resistance above 5 cps (see table 2).) At high frequencies the largest value of $R_{2}$ employed is $10^{8} \mathrm{ohms}$. Therefore, the largest value of $C_{s}^{\prime} R_{2} F$ ever encountered is 0.15 .

The correction term contains the multiplying factor $\left(1+\omega^{2} C_{4} C_{g} J_{1}^{2} / 2\right)$. Setting $C_{4}$ equal to $1450 \mathrm{pf} \pm$ 10 percent (this value is determined in appendix 7.4), then $\omega^{2} C_{4} C_{g} J_{1}^{2} / 2$ becomes $(0.286 \pm 0.029)$ at 200 cps. Therefore, the total correction becomes $(0.15+$ $0.043 \pm 0.004)$.

\subsection{Bridge Equations with $\mathrm{C}_{g}$ Shorted}

Let us now consider the case in which $C_{g}$ is shorted $\left(C_{g}=\infty\right)$, the mode of operation to be used in practice. With $C_{\mathrm{g}}$ shorted, $J_{1}$ and $J_{2}$ are in parallel with $R_{3}$ and $R_{A}$ respectively. This lowers the equivalent resistance of each arm, and furthermore makes the equivalent resistance independent of frequency.

The exact bridge equations for the case $C_{g}=\infty$ are given in appendix 7.3. Simplified equations which give $C_{x}$ and $G_{x}$ to a sufficient accuracy are

$$
\begin{aligned}
& C_{x}=\Delta C_{s}+C_{s}^{\prime} \frac{\Delta R_{1}}{R_{4}} K+\left(C_{3}-C_{4}\right) \frac{\Delta R_{1}}{R_{2}} K, \\
& G_{x}=\frac{\Delta R_{1}}{R_{2} R_{4}}\left[1+C_{s}^{\prime} R_{2} \omega^{2} C_{4} R_{4} K^{2}\right],
\end{aligned}
$$

where

$$
K=J_{2} /\left(J_{2}+R_{4}\right)=0.136 .
$$

Notice that one of the correction terms extant in eq (10) is absent in eq (22). Both of the correction terms of eq (22) contain the factor $K$, which now has the constant value 0.136 , whereas in eq (10), $K$ varied with frequency having a maximum value of unity. In eq (22) the correction term involving $\left(C_{3}-C_{4}\right)$ may be made negligibly small by balancing $C_{3}$ and $C_{4}$ at $200 \mathrm{cps}$ (see eq (21)). The term $C_{s}^{\prime} K \Delta R_{1}$ $R_{4}$ now has a maximum value of only 0.019 pf.

The expression for the conductance, eq (23), contains a small frequency-dependent correction term. This term is largest when $C_{s}^{\prime}=100 \mathrm{pf} ; R_{2}=10^{8} \mathrm{ohms}$, and $f=200 \mathrm{cps}$. Using $C_{4}=1450 \mathrm{pf} \pm 10$ percent, the term becomes $0.05 \pm 0.005$. This value is much less than its counterpart in eq (11), which is 0.19. It should be noted that the 10 percent uncertainty in $C_{4}$ may cause an error of 1 percent or more at frequencies above $200 \mathrm{cps}$.

Below 9 cps, even if $R_{2}$ were $10^{10} \mathrm{ohms}$, the correction term of eq (23) contributes less than one percent to the total conductance and, therefore, may be ignored.

Since the correction terms in eqs (22) and (23) are smaller and far less dependent upon frequency than those in eqs (10) and (11), in practice the bridge is operated with $C_{g}=\infty$ where ecs (22) and (23) apply.
The reader is reminded that eqs (22) and (23) apply to substitution measurements only if $C_{3}$ is decreased by $C_{x}$ when the unknown is switched "out." The procedure is outlined in section 3.2.

Inspection of eqs (22) and (23) shows that capacitances between zero and 100 pf are measurable, while conductances between $10^{-9}$ and $10^{-16}$ mhos may possibly be measured. The sensitivity of the apparatus, however, places closer limits on these extremes, particularly upon the lower limit of conductance.

\subsection{Sensitivity}

It is informative to calculate the magnitude of the voltage appearing across the detector terminals due to a small departure from perfect capacitive or resistive balance. The expression for this voltage shows how to improve the sensitivity and predicts the smallest observable impedance unbalance.

The simplified circuit diagram in figure 4 is used in calculating the sensitivity. With $C_{g}$ shorted, the equivalent resistances of the ratio arms are denoted as $R_{K}$ and $R_{L}$. The impedance, $Z_{D}$, which shunts the detector terminals, consists of the input impedance of the detector employed $\left(R_{I}\right)$, paralleled by $R_{E}$, as given in eq (1), and by the total capacitance to ground across the detector terminals, $C_{D}$. The expression for $1 / Z_{D}$ is

$$
\frac{1}{Z_{D}}=\frac{1}{R_{D}}+j \omega C_{D},
$$

where $R_{D}$ is the resultant parallel resistance of $R_{E}$ and $R_{I}$.

The voltage developed across the detector terminals, $V_{D}$, is given by

$$
V_{D}=\frac{V\left[R_{L} / Z_{P}-R_{K} / Z_{B}\right]}{\left(R_{L}+R_{K}\right)\left(\frac{1}{Z_{D}}+\frac{1}{Z_{P}}+\frac{1}{Z_{B}}\right)+\frac{R_{L} R_{K}}{Z_{P}}\left(\frac{1}{Z_{P}}+\frac{1}{Z_{B}}\right)},
$$

where $V$ is the applied voltage.

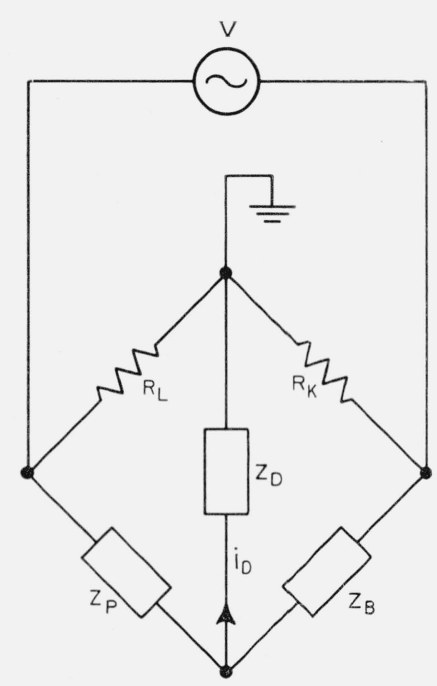

FiguRE 4. Simplified circuit diagram from which the sensitivity $i$ s calculated. 
We now make the approximations that $R_{L}=R_{K}$, and that the bridge is sufficiently balanced so that we may set $Z_{P}$ equal to $Z_{B}$ in the denominator of eq (25). Equation (25) becomes

$$
V_{D}=\frac{\frac{V}{2}\left(\frac{1}{Z_{P}}-\frac{1}{Z_{B}}\right)}{\frac{1}{Z_{D}}+\frac{2}{Z_{B}}+\frac{R_{L}}{Z_{D} Z_{B}}} .
$$

The term $R_{L} / Z_{B} Z_{D}$ may be dropped from the denominator leaving

$$
V_{D}=\frac{\frac{V}{2}\left[\frac{1}{Z_{P}}-\frac{1}{Z_{B}}\right]}{\frac{1}{Z_{D}}+\frac{2}{Z_{B}}}
$$

Substituting for the $Z$ 's and using the approximation that $2 / R_{B} \ll 1 / R_{D}$ we obtain

$V_{D}=$

$\frac{(V / 2) R_{D}\left[1 / R_{P}-1 / R_{B}+j \omega\left(C_{P}-C_{B}\right)\right]\left[1-j \omega R_{D}\left(2 C_{B}+C_{D}\right)\right]}{1+\omega^{2} R_{D}^{2}\left(2 C_{B}+C_{D}\right)^{2}}$

This approximate equation predicts that the bridge is balanced ( $V_{D}=$ zero) when $R_{P}=R_{B}$ and $C_{P}=C_{B}$.

Let a small departure from capacitive balance be represented by $\pm \delta C_{P}$ such that

$$
C_{P}-C_{B}= \pm \delta C_{P}
$$

A small conductance unbalance, $\delta G_{P}$, is defined as

$$
1 / R_{P}-1 / R_{B}= \pm \delta G_{P}
$$

Equation (28) may be used to calculate $V_{D}$ when a slight conductance unbalance exists in the presence of perfect capacitive balance. Setting $\delta C_{P}=0$, and using eq (30) one obtains for the amplitude of $V_{D}$.

$$
\left|V_{D}\right|=\frac{\left|\frac{V}{2}\right| R_{D}\left|\delta G_{P}\right|}{\sqrt{1+\omega^{2} R_{D}^{2}\left(2 C_{B}+C_{D}\right)^{2}}} .
$$

Therefore,

$$
\left|\frac{V_{D}}{\delta G_{P}}\right|=\frac{\left|\frac{V}{2}\right| R_{D}}{\sqrt{1+\omega^{2} R_{D}^{2}\left(2 C_{B}+C_{D}\right)^{2}}} .
$$

The expression for $V_{D}$ per unit capacitance unbalance in the presence of perfect resistive balance may similarly be derived and is

$$
\left|\frac{V_{D}}{\delta C_{P}}\right|=\frac{\left|\frac{V}{2}\right| \omega R_{D}}{\sqrt{1+\omega^{2} R_{D}^{2}\left(2 C_{B}+C_{D}\right)^{2}}} .
$$

Equations (32) and (33) both show that the sensitivity may be increased by lowering $C_{D}$. This may be accomplished by minimizing the appropriate lead capacitances and the input capacitance of the detecting device. ${ }^{8}$

In figure 5 is plotted $V_{D} / \delta C_{P}{ }^{9}$ versus $\left(f R_{D}\right)$. Here we have estimated $C_{D}=200 \mathrm{pf}$, and assumed $C_{B}=60$ pf. The peak-to-peak voltage of the generator, $V$, is 30 volts. Note that in those cases in which $\omega R_{D}$ $\left(2 C_{B}+C_{D}\right)<1, V_{D} / \delta C_{P}$ varies linearly with the product $\left(\omega R_{D}\right)$. Under these conditions, the sensitivity may be held roughly constant as $\omega$ decreases by increasing $R_{D}$ by a multiple of ten for each decade decreased in $\omega$. Since $1 / R_{D} \cong 1 / R_{2}+1 / R_{I}, R_{D}$ can be increased by increasing $R_{2}$, but can never become greater than $R_{I}$. Therefore, the value of the input resistor of the detector will ultimately limit the sensitivity at low frequencies. Also, since the conductance must be balanced, one is not entirely free in choice of $R_{2}$. Optimum conditions exist when $R_{2}$ is made as large as possible while still permitting conductance balance of the bridge. In general the smaller $R_{x}$, the poorer the sensitivity to capacitive unbalance.

When $\omega R_{D}\left(2 C_{B}+C_{D}\right)>1, \quad V_{D} / \delta C_{P}$ approaches a maximum of

$$
\frac{V_{D}}{0.001 \mathrm{pf}}=\frac{\frac{V}{2} \times 10^{-15}}{2 C_{B}+C_{D}}=4.7 \times 10^{-5} \frac{\text { peak-to-peak volts }}{0.001 \mathrm{pf}} .
$$

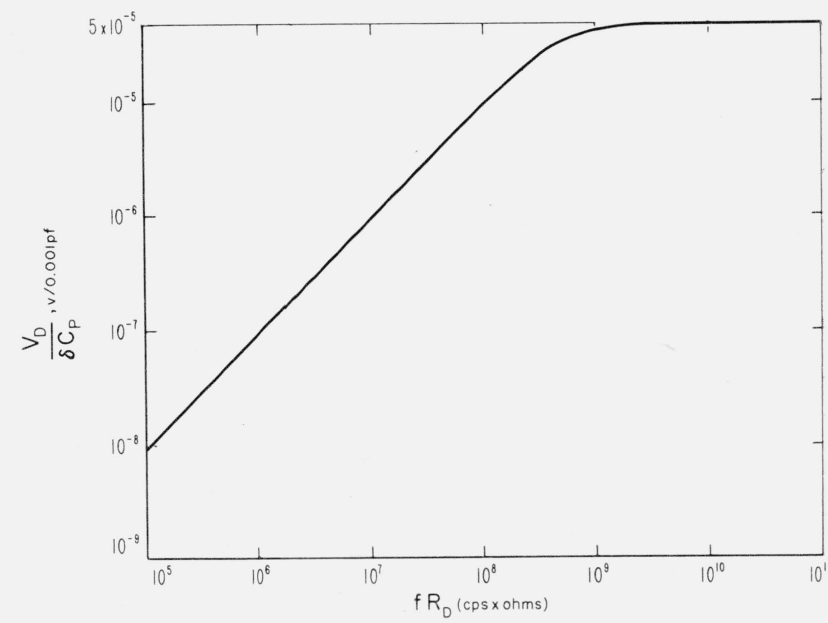

FiguRe 5. Log-log plot of the voltage appearing across the detector terminals $\left(\mathrm{V}_{\mathrm{D}}\right)$ per increment capacitance unbalance $\left(\delta \mathrm{C}_{\mathrm{P}}\right)$ versus the product $\mathrm{fR}_{\mathrm{D}}$.

In figure 6 is plotted $V_{D} / \delta G_{P}$ vs. frequency for various values of $R_{D}$. The smallest increment in $G_{P}$ possible with this apparatus $\left(10^{-16} \mathrm{mhos}\right)$ is chosen for $\delta G_{P}$. Note that when $\omega R_{D}\left(2 C_{B}+C_{D}\right)>1, V_{D} / \delta G_{P}$

8 In order to lower $C_{D}$ the 250 pf capacitor shunting the input resistor of the Ultrohmeter has been disconnected.

9 The amplitude symbol, | |, is dropped at this point. 
increases as the frequency decreases and is independent of $R_{D}$. When $\omega R_{D}\left(2 C_{B}+C_{D}\right)<1, V_{D} / \delta G_{P}$ is independent of frequency but increases linearly with $R_{D}$. In general, best sensitivity to conductance unbalance is attained at low frequency using high values of $R_{D}$.

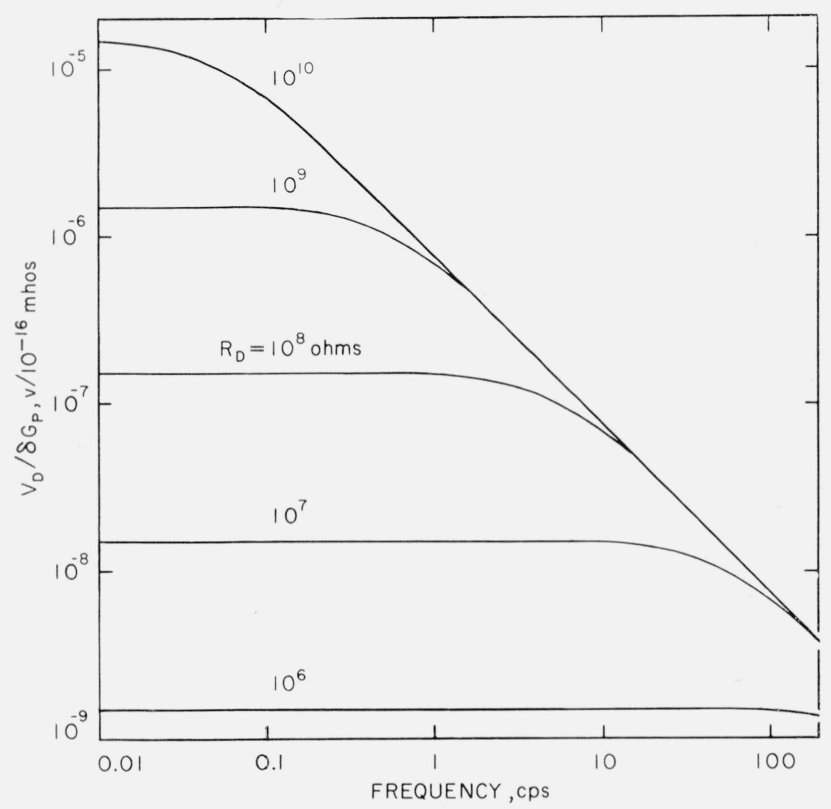

FIgURE 6. The unbalance voltage per increment conductance unbalance versus frequency.

Once the sensitivity of the detector employed is ascertained, this graph may be used to determine the minimum detectable conductance unbalance under various operating conditions,

In practice the bridge is operated using the highest value of $R_{2}{ }^{10}$ that will permit resistive balance. This condition also enhances $V_{D} / \delta C_{P}$, as was previously discussed.

Equations (32) and (33) give the voltage at the output terminals for a given bridge unbalance. Whether or not this voltage is observable depends unpon the sensitivity of the detector. If we denote minimum voltage detectable as $v_{m}$, then the smallest capacitive unbalance detectable becomes

$$
d C=\frac{v_{m}}{V_{D} / \delta C_{P}} \cdot
$$

The smallest detectable conductance unbalance is

$$
d G=\frac{v_{m}}{V_{D} / \delta G_{P}} .
$$

The accuracy with which $d C$ and $d G$ may be computed depends upon the accuracy with which $v_{m}$ and

10 Maximizing $R_{2}$ or equivalently $\Delta R_{1}$, will also increase the magnitude of the correction term, $C_{s}{ }^{\prime} K \Delta R_{1} / R_{4}$ which appears in eq $(22)$.
$C_{D}$ are known. It is impossible to determine $v$ with great precision. Also the value of $C_{D}$ employed (200 pf), is merely an estimate. Therefore, values of $d C$ and $d G$ computed from eqs (34) and (35) may be in error by as much as 100 percent.

Calculated values of $d C$ and $d G$, for various possible conditions of operation, are shown in table 2 . These calculated values have proven to be essentially correct experimentally. Since the sensitivity, $v_{m}$, of the detector employed, ${ }^{11}$ as well as its input resistance, $R_{I}$, effect the results, the values of $d C$ and $d G$ will be different if other detection systems are employed.

TABLE 2. The minimum detectable capacitance unbalance, $d C$, and minimum detectable conductance unbalance, $d G$. for various oper-

\begin{tabular}{|c|c|c|c|c|c|}
\hline $\begin{array}{l}\text { Frequen- } \\
\text { cy }\end{array}$ & $\begin{array}{c}\text { Detec- } \\
\text { tor a }\end{array}$ & $R_{2}$ & $d C$ & $d G$ & $G_{m}^{\mathrm{b}}$ \\
\hline \multirow{3}{*}{$\begin{array}{l}c p s \\
100\end{array}$} & & ohms & $p f$ & mhos & mhos \\
\hline & I & $10^{6}$ & $<0.001$ & 1. $3 \times 10^{-13}$ & $10^{-12}$ \\
\hline & & $10^{8}$ & $<.001$ & 2. $6 \times 10^{-14}$ & $10^{-14}$ \\
\hline \multirow[t]{2}{*}{10} & I & $10^{6}$ & .002 & 1. $3 \times 10^{-13}$ & $10^{-12}$ \\
\hline & & $10^{8}$ & .001 & 1. $3 \times 10^{-14}$ & $10^{-14}$ \\
\hline \multirow[t]{2}{*}{1} & II & $10^{6}$ & & 1. $3 \times 10^{-12}$ & $10^{-12}$ \\
\hline & & $10^{10}$ & .001 & 2. $6 \times 10^{-15}$ & $10^{-16}$ \\
\hline \multirow[t]{2}{*}{0.1} & II & $10^{6}$ & 2. 0 & 1. $3 \times 10^{-12}$ & $10^{-12}$ \\
\hline & & $10^{10}$ & $<0.001$ & 3. $0 \times 10^{-16}$ & $10^{-16}$ \\
\hline \multirow[t]{2}{*}{0.01} & III & $10^{6}$ & 20.0 & 1. $3 \times 10^{-12}$ & $10^{-12}$ \\
\hline & & $10^{10}$ & 0.002 & 1. $3 \times 10^{-16}$ & $10^{-16}$ \\
\hline
\end{tabular}
ating conditions.

a Detector I-preamplifier-waveanalyzer, II-Ultrohmeter, III-Ultrohmeterd-c recorder.

b $G_{m}$ is the conductance change due to a $0.1 \mathrm{ohm}$ change in $\mathrm{R}_{1}$.

Between 200 and 5 cps capacitance unbalances of $0.001 \mathrm{pf}$ may almost always be detected.

With the Ultrohmeter as detector between 2 and $0.1 \mathrm{cps}, d C$ is less than 0.001 pf unless $f R_{D}<2 \times 10^{8}$ cps-ohm. Then $d C$ is given approximately by

$$
d C=\frac{2 \times 10^{5}}{f R_{D}}(\mathrm{pf}) .
$$

At 1 cps with $R_{2}=10^{6}$ ohms, $d C$ is 0.2 pf. At 0.1 cps with $R_{2}=10^{6}$ ohms, $d C$ is $2 \mathrm{pf}$, which is a large uncertainty. Fortunately, the situation rarely arises which requires $R_{2}=10^{6} \mathrm{ohms}$ at $0.1 \mathrm{cps}$. Most dielectrics have a loss index, $\epsilon^{\prime \prime}$ less than unity. Since

$$
\epsilon^{\prime \prime}=\frac{1}{\omega R_{x} C_{v}^{\prime}},
$$

11 The effective sensitivities of the detectors employed are somewhat frequency dependent, as well as functions of the noise level. At 100 through 5 cps an error dependent, as well as functions of the noise level. At 100 through 5 cps an error voltage of about $2 \times 10^{-6}$ volts can be detected. A signal of about $2 \times 10^{-5}$ volts
can be detected on the Ultrohmeter at $2 \mathrm{cps}$ and at $0.1 \mathrm{cps}$. At intermediate frequencies, signals as low as $3 \times 10^{-6}$ volts are observable. A d-c voltage of $4 \times 10^{-}$ volts, however, just gives a detectable indication (about one tenth division deflection). The increased sensitivity of the Ultrohmeter to a low frequency a-c signal is possibly due to the feedback utilized, or to a mechanical "resonance" of the meter's pointer. Using the Ultrohmeter-recorder system below $0.1 \mathrm{cps}$ a signal of $2 \times 10^{-5}$ volts is detectable. 
if the specimen's equivalent vacuum capacitance, $C_{v}$, were about $10 \mathrm{pf}$, its loss index would have to be near 100 to require use of the $10^{6} \mathrm{ohm}$ value of $R_{2}$ at $0.1 \mathrm{cps}$. Similarly, at $0.01 \mathrm{cps}$, the $10^{6}$ or even the $10^{7} \mathrm{ohm}$ range would practically never be required.

The minimum conductance detectable throughout the frequency range is given roughly by the approximate expression

or

$$
G_{x m}=3 f \times 10^{-15} \text { mhos when } \mathrm{f} \geq 0.1 \mathrm{cps}
$$

$$
G_{x m}=2 \times 10^{-16} \text { mhos when } \mathrm{f}<0.1 \mathrm{cps}
$$

(This expression underrates the capabilities of the bridge above 5 cps.)

The minimum detectable loss index is approximately,

or

$$
\epsilon_{m}^{\prime \prime}=5 \times 10^{-4} / C_{v}(\mathrm{pf}) \text { when } f \geq 0.1 \mathrm{cps}
$$

$$
\epsilon_{m}^{\prime \prime}=3 \times 10^{-5} / f C_{v}(\mathrm{pf}) \text { when } f<0.1 \mathrm{cps} \text {. }
$$

\section{Experimental Check of the Bridge}

In order to check the bridge experimentally, measurements were made upon resistors and capacitors of known values. Most of these measurements were performed both with $C_{g}=1 \mu$ f and with $C_{g}=\infty$. Comparison of the results obtained using the two methods in no case revealed any significant discrepancies.

\subsection{Measurements upon Capacitors and Resistors of Known Values}

Preliminary measurements were made upon a 5.004 pf fixed reference standard capacitor in parallel with resistors ranging in value from $10^{10}$ to $10^{14}$ ohms. The measured value of the parallel capacitance of the resistor itself was subtracted from the measured capacitance of the $R C$ combination to obtain values associated with the standard capacitor. The results of some thirty measurements made at various frequencies within the range of $0.1 \mathrm{cps}$ to 200 cps, averaged to 5.0055 pf with a standard deviation of $0.0013 \mathrm{pf}$. This agreement is satisfactory, the error being only 0.03 percent.

The results of measurements on resistors are shown in table $3 .^{12}$ The d-c values given in the last row were determined to an accuracy of better than 0.5 percent by an independent technique due to Scott [8] in which a variable air capacitor is discharged through the unknown resistor while holding the voltage constant by smoothly decreasing the capacitance. The values obtained at low frequencies agree with the d-c values to well within 1 percent for the $10^{9}, 10^{10}, 10^{11}, 10^{12}$, and $10^{13} \mathrm{ohm}$ resistors. This clearly shows the bridge's ability to accurately measure resistances in this range at low frequencies. At high frequencies however, there is extreme departure from the $d-c$ values for nearly all the resistors. Rough measurements made with a twoterminal Schering bridge near 100 cps essentially corroborate the findings of the low frequency bridge in this region. These results lead to the conclusion that the particular composition resistors measured exhibited a strong frequency dependence.

2 These resistors were measured in a shielded chamber through which a flow of dried nitrogen gas was maintained. Measurements on the $10^{13} \mathrm{ohm}$ resistor of dried nitrogen gas was maintained. Measurements on the $10^{13} \mathrm{ohm}$ resistor higher than it was with dry nitrogen flowing. Results below to be a decade higher than it was with dry nitrogen flowing. Results below 1 cps were un-
affected by this humidity change. Since we have no assurance that the flow of

\begin{tabular}{|c|c|c|c|c|c|c|}
\hline \multirow{2}{*}{ Frequency } & \multicolumn{6}{|c|}{ Measured value in ohms } \\
\hline & Resistor 1 & Resistor 2 & Resistor 3 & Resistor 4 & Resistor 5 & Resistor 6 \\
\hline $\begin{array}{l}c p s \\
100\end{array}$ & & & & & & \\
\hline 100 & 1. $125 \times 10^{9}$ & $0.868 \times 10^{10}$ & $0.563 \times 10^{11}$ & $0.097 \times 10^{12}$ & $0.087 \times 10^{13}$ & $0.013 \times 10^{14}$ \\
\hline $\begin{array}{l}50 \\
20\end{array}$ & 1. 160 & .895 & .678 & .214 & .104 & .021 \\
\hline 20 & 1. 167 & .949 & .803 & .427 & & .037 \\
\hline $\begin{array}{r}10 \\
5\end{array}$ & 1. 169 & .960 & $\begin{array}{l}.874 \\
.942\end{array}$ & $\begin{array}{l}.59 \\
.75\end{array}$ & $\begin{array}{l}.258 \\
.321\end{array}$ & .062 \\
\hline 2 & 1. 173 & .980 & 980 & .87 & .420 & .120 \\
\hline 1 & 1. 173 & .983 & .990 & .95 & .54 & 160 \\
\hline 0.5 & 1. 174 & .983 & 994 & .98 & .64 & .211 \\
\hline .2 & 1. 174 & .983 & .999 & 1. 002 & .78 & \\
\hline $\begin{array}{l}.1 \\
.05\end{array}$ & 1. 173 & .983 & 1. 000 & $\begin{array}{l}\text { 1. } 004 \\
\text { 1. } 004\end{array}$ & $\begin{array}{l}.88 \\
.92\end{array}$ & $\begin{array}{l}.35 \\
.46\end{array}$ \\
\hline .01 & (b) & & & 1. 006 & 1. 00 & .84 \\
\hline$d-c(a)$ & 1. $173 \times 10^{9}$ & $.9835 \times 10^{10}$ & $.995 \times 10^{11}$ & 1. $002 \times 10^{12}$ & 1. $01 \times 10^{13}$ & 1. $11 \times 10^{14}$ \\
\hline
\end{tabular}
affected by this humidity change. Since we have no assurance that the flow of nitrogen removed all surface moisture, it is possible that
at high frequencies may be partially due to moisture.

TABLE 3. Results of measurements on resistors

a These $d-c$ values are essentially independent of voltage up to 15 volts.

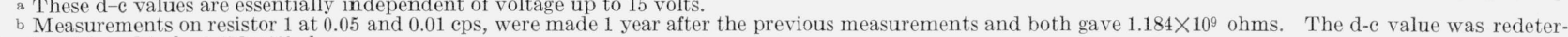
mined and found to be $1.178 \times 10^{9} \mathrm{ohms}$. 
This frequency dependence is understandable in light of the theoretical work of Howe [9]. He considered a cylindrically shaped composition resistor and associated with it a constant capacitance per unit length. He showed that the equivalent parallel resistance and capacitance drop off sharply from their d-c values as the frequency is increased above a certain value. From his work, it can be inferred that the equivalent resistanee has decreased about one percent from its d-c value $(R)$ when the product $(R f)$ is near $10^{12}$ cps-ohms, and decreases sharply at higher frequencies. Using this rule, a $10^{12} \mathrm{ohm}$ resistor should begin to exhibit a frequency dependence near $1 \mathrm{cps}$, a $10^{13} \mathrm{ohm}$ resistor near $0.1 \mathrm{cps}$, etc. Referring to table 3, we see that these predictions are roughly verified.

The frequency dependence of the nominal $10^{14}$ ohm resistor was further investigated using d-c techniques. The current in the resistor was measured as a function of time, $t$, after application of 20 volts d-c. The resistance at time $t$ was then calculated using Ohm's law. A frequency, $f$, was associated with the time interval, $t$, according to the equation:

$$
f=0.1 / t \text {. }
$$

Equation (40) has been proposed and used by Hamon [10]. He has shown that under certain conditions eq (40) may be used to convert from decay current studies of dielectrics to their a-c dielectric properties. Although there is no assurance that the conditions which justify the use of eq (40) are rigorously fulfilled in the present experiment, it is the only such equation which has been justified and it is employed here with reasonable success.

The results of these d-c measurements on resistor 6 are shown in figure 7 along with the results obtained on the low frequency bridge. The two sets of data form a reasonably smooth curve, which gives additional confidence in the accuracy of the low frequency bridge in measurements of high values of resistance. The d-c measurements also provide an independent demonstration of the frequency dependence of these resistors.

The frequency dependence of these resistors makes it impossible to present a clear cut demonstration of the accuracy of the bridge through measurements of fixed, known, conductances across its entire frequency range. Estimates of the accuracy of conductance measurements is based therefore upon (a) sensitivity considerations (eq (38)), (b) the fact that measurements at low frequencies on lower values of resistance are within one percent of the d-c value, and (c) the agreement, at higher frequencies, with Schering bridge measurements (see section $5)$. The overall accuracy in conductance is therefore about $\pm\left(1\right.$ percent $+3 f \times 10^{-15}$ mhos $)$ when $f \geq 0.1 \mathrm{cps}$, and about \pm ( 1 percent $+2 \times 10^{-16}$ mhos) when $\mathrm{f}<0.1 \mathrm{cps}$.

From similar evidence the accuracy of capacitance measurements may be estimated. While using the preamplifier-wave analyzer as detector, capacitances can be measured to an accuracy of about $\pm(0.05$

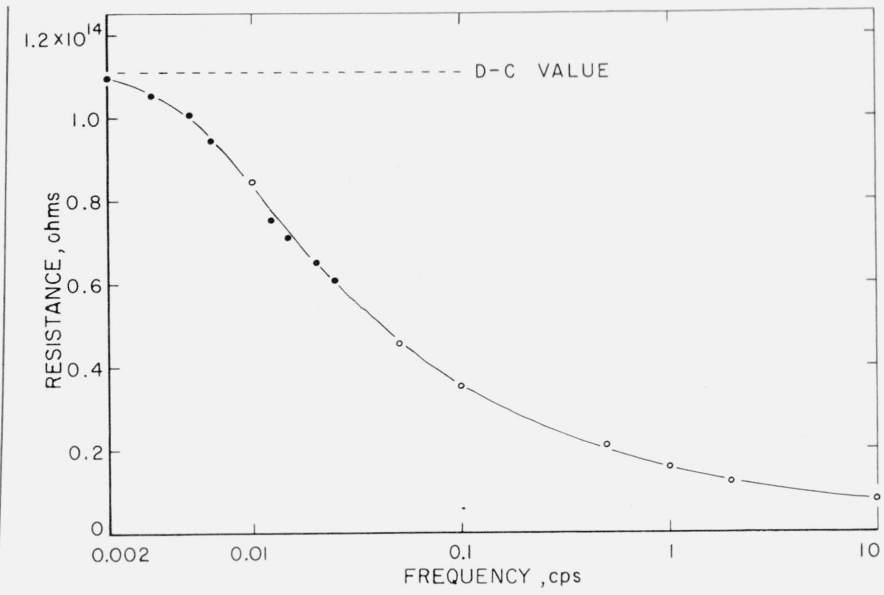

FiguRe 7. Frequency dependence of a nominal $10^{14} \mathrm{ohm}$ resistor (resistor 6).

-low frequency apparatus

- d-c decay measurement using $f=0.1 / t$, where $t$ is the time in seconds after application of the $\mathrm{d}-\mathrm{c}$ voltage.

percent $+0.002 \mathrm{pf}$ ). Using the Ultrohmeter below 5 cps the accuracy is about $\pm[0.05$ percent $+(0.002$ $\left.\left.+2 \times 10^{5} / f R_{D}\right) \mathrm{pf}\right]$.

\subsection{The Effects of $Z_{1}, Z_{2}$, and $Z_{L}$}

Let us now investigate the effects of the stray impedances to ground within the generator $Z_{1}$, $Z_{2}$, and $Z_{L}$. Referring to figures 1 and 2 it is apparent that any of these impedances may give rise to resistive and (or) capacitive shunts across $R_{3}$ and $R_{A}$. The possibility exists that these shunts may be frequency dependent. The bridge equations, (10) and (11), show that impedances shunting $R_{3}$ or $R_{A}$ affect the results of substitution measurements only in the "correction terms". Therefore, it is possible to learn something of the magnitude of these stray impedances through an experimental study of these terms.

\section{a. Stray Resistive Shunting of the Ratio Arms}

The largest of the correction terms in eq (10) is the quantity, $C_{s}^{\prime} K \Delta R_{1} / R_{4}$, which has a maximum value of $0.138 \mathrm{pf}$. Through an experimental study of this term it is demonstrated that no appreciable stray resistive shunting of the ratio arms occurs. An understanding of the origin of this term will be helpful in the discussion to follow.

The correction term, $\left(C_{s}^{\prime} \Delta R_{1} K / R_{4}\right)$, arises from the fact that the equivalent value of one of the ratio arms is dependent upon the setting of $R_{1}$. Since $R_{1}$ is varied to balance the conductance, $R_{A}$ will be different for the two bridge balances necessary in the substitution method. If the ratio arms are not the same at the two balances, the capacitance measurement requires a correction. 
It can be shown that if any resistor, which we shall call $J_{K}$ shunts $R_{A}$ (such as $J_{2}$ shunts $R_{A}$ when $C_{g}$ is shorted $)^{13}$ then $K$ is given by

$$
K=\frac{J_{K}}{J_{K}+R_{4}} .
$$

Therefore, through measurements of $K$ it is possible not only to ascertain that a resistor is shunting $R_{A}$, but also to determine its magnitude.

The magnitude of $K$ can be determined by performing two substitution measurements of the parallel capacitance and resistance of a resistor. These measurements are made using two consecutive values of $R_{2}$. Using values of $R_{2}$ having a ratio of ten to one necessitates that the ratio of the $\Delta R_{1}$ 's between the two measurements be one to ten. Therefore, the magnitudes of $C_{s}^{\prime} \Delta R_{1} K / R_{4}$, will differ by nearly a multiple of ten. The difference in $\Delta C_{s}$ and in $\Delta R_{1}$ between the two measurements (labeled 1 and $2)$ are $\left[\left(\Delta C_{s}\right)_{1}-\left(\Delta C_{s}\right)_{2}\right]$ and $\left[\left(\Delta R_{1}\right)_{1}-\left(\Delta R_{1}\right)_{2}\right]$ respectively. $K$ is then

$$
K=\frac{R_{4}\left[\left(\Delta C_{s}\right)_{1}-\left(\Delta C_{s}\right)_{2}\right]}{C_{s}^{\prime}\left[\left(\Delta R_{1}\right)_{2}-\left(\Delta R_{1}\right)_{1}\right]}
$$

The preceding outlines the method used to detect a resistive shunt on $R_{A}$. Due to the symmetry of the generator circuit one would expect that if a resistance shunts $R_{A}$, one nearly equal in magnitude would shunt $R_{3}$. One cannot be certain of this, however, and a method through which a resistive shunt on $R_{3}$ may be detected must be devised. The method employed involves observing the change in capacitive balance when the generator terminals are interchanged. At balance the ratio of the capacitances will be determined essentially by the ratio of the resistive arms. If $R_{3}$ and $R_{A}$ are asymmetrically shunted, upon interchange of the generator terminals the capacitive balance will change. With the generator connected as originally shown in figure 1 (i.e., $J_{1}$ connected to point $W$ in the bridge), the value of $C_{s}$ at balance will be called $C_{s o}$. The value of $C_{s}$ at balance after interchanging the generator connections (with $C_{B}$ unchanged) is called $C_{s i}$. From eq (6) with $C_{g}=\infty, J_{1}=J_{L}$, and $J_{2}=J_{K}$, the ratio of the resistors shunting $R_{3}$ and $R_{A}$ is given to a close approximation by

$$
\frac{J_{L}}{J_{K}}=1-\frac{\left(C_{s o}-C_{s i}\right)}{2 C_{B}}\left(1+\frac{J_{L}}{R_{A}}\right),
$$

where $J_{L}$ represents the resistance shunting $R_{3}$ in the original bridge-generator connection. The departure of $J_{L} / J_{K}$ from unity is therefore directly proportional to $\left(C_{s o}-C_{s i}\right) / 2 C_{B}$. Thus, measurements of $\left(C_{s o}-C_{s i}\right)$ and of $K$ are sufficient to determine $J_{K}$ and $J_{L}$.

13 Even with $C_{g}=1 \mu \mathrm{f}$ at frequencies near $100 \mathrm{eps} K$ is $J_{2} /\left(J_{2}+R\right)=0.136$. This means that the capacitive coupling through $\mathrm{C}_{g}$ is sufficient to effectively shunt $R_{A}$ by $J_{2}$. Near 0.1 cps, however, the value of $K$ is unity, implying that this capacitive coupling is no longer effective. At about $3 \mathrm{cps}$, where $Z_{g} \cong 10^{5} \mathrm{ohms}$, the "crossover" between these two extremes occurs.
The results of these experiments are shown in figures 3 and 8 . In figure 3 the curves are calculated from eq (13), which gives $K$ as a function of frequency. The experimental points fall on the calculated curves to within the experimental error. Note that with $C_{g}=1 \mu \mathrm{f}, K$ is unity near $0.1 \mathrm{cps}$, indicating that there is no effective resistive shunt across $R_{A}$. Near $100 \mathrm{cps}, K$ becomes essentially 0.136 as predicted. With $C_{g}=\infty, K$ remains near 0.136 , indicating a frequency-independent $15.8 \mathrm{kohm}$ shunt.

At frequencies near $100 \mathrm{cps}$, the value of $K$ was also experimentally determined with $C_{g}=0.1 \mu \mathrm{f}$ and $0.01 \mu \mathrm{f}$. This was done to obtain a more sensitive test for stray resistive shunting at high frequencies. These points also fall on the calculated curves to within the experimental error. Therefore up to 100 cps the stray impedances within the generator aive rise to no significant resistive shunt on $R_{A}$. Even a $10^{6} \mathrm{ohm}$ shunt across $R_{A}$ will have no noticeable effect upon the results if the substitution method is employed, particularly if one operates the bridge with $C_{g}=\infty$.

Figure 8 gives the results of measurements of the change in capacitance balance, upon interchange of the generator-to-bridge connections. The experimental points for $C_{g}=0$ indicate that no asymmetric resistive shunting exists up to a frequency of 100 cps. Above $100 \mathrm{cps}$ such shunting is evident with $C_{g}=0$. With $C_{g}=1 \mu \mathrm{f}$ or $C_{g}=\infty$, the effect of this asymmetric shunt is lessened, and becomes evident only above $200 \mathrm{cps}$.

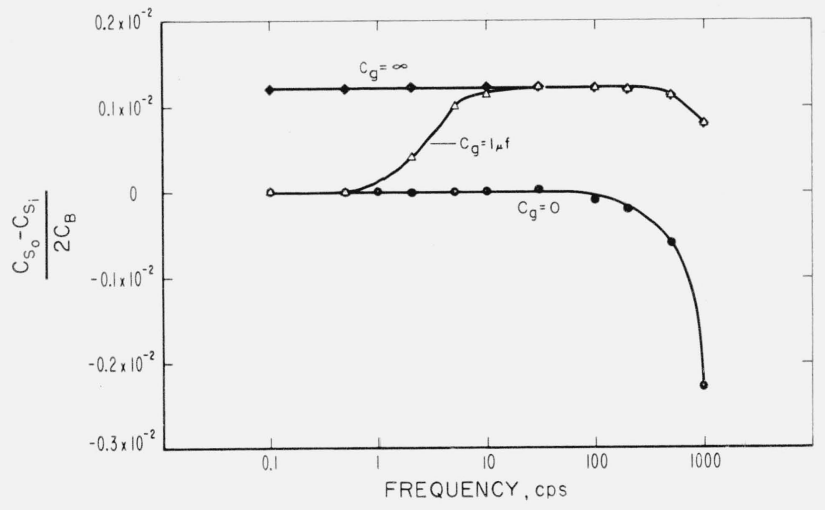

FiguRE 8 . The change in capacitance balance $\left(\mathrm{C}_{\mathbf{s}_{\mathrm{o}}}-\mathrm{C}_{\mathrm{si}_{\mathrm{i}}}\right)$ upon interchange of the bridge-generator connections.

The change in capacitance balance with interchange of the generator to bridge connection, according to eq (43), serves as a measure of the unbalance of resistive shunts arising within the generator and placed across $R_{3}$ and $R_{A}$.

Below 200 cps, with $C_{g}=\infty$, the value of $\left(C_{s o}-C_{s i}\right) / 2 C_{B}$, is independent of frequency and is 0.00122 . From this one may compute the value of $r$ by using eq (43). Substituting we have:

$$
r=\frac{J_{1}}{J_{2}}=1-0.00122\left(1+\frac{1.58 \times 10^{4}}{10^{5}}\right),
$$

giving $r=0.99859 .^{14}$

14 The ratio $r$ was also determined by an independent d-c technique which gave $r=0.99860 \pm .00005$. 
The experimental points for $C_{g}=1 \mu$ f clearly show the "crossover" between asymmetric shunting by $J_{1}$ and $J_{2}$ at high frequencies, and no shunting at low frequencies.

In summary, it has been shown, through measurements of $K$ and $\left(C_{s o}-C_{s i}\right) / 2 C_{B}$, that the resistance shunting the bridge arms due to the stray impedances in the generator, is most certainly greater than $10^{6} \mathrm{ohms}$, and that below $100 \mathrm{cps}$ such resistive shunting that may exist is symmetrical to within the requirements of this apparatus.

\section{b. Capacitive Shunts across the Ratio Arms}

Any capacitive shunt originating from the stray generator impedances $Z_{1}, Z_{2}$, and $Z_{L}$ and placed across $R_{3}$ and $R_{A}$ are included in the total capacitance across these arms, $C_{3}$ and $C_{4}$ respectively. In appendix 7.4 the value of $C_{4}$ is found to be about $1400 \mathrm{pf}$ at 100 cps. It is necessary to know $C_{4}$ in order to evaluate the correction term in the conductance equation (eq (23)). It is shown in section 3.2a, eq (21) that $\left(C_{3}-C_{4}\right)$ can be made zero at $100 \mathrm{cps}$ by adjusting the variable capacitor across $R_{3}$. This adjustment eliminates, at high frequencies, a correction term involving $\left(C_{3}-C_{4}\right)$ from the capacitance equation (eq (22)). However, since $C_{3}$ and $C_{4}$ contain components which are possibly frequency dependent, it is necessary to verify that $\left(C_{3}-C_{4}\right)$ remains zero at lower frequencies.

An attempt was made to determine $\left(C_{3}-C_{4}\right)$ at $0.5 \mathrm{cps}$ by measuring the magnitude of the correction term $\left(C_{3}-C_{4}\right) K \Delta R_{1} / R_{2}$ in eq (10). At $0.5 \mathrm{cps}$, the sensitivity of the Ultrohmeter is best, and with $C_{g}=1 \mu \mathrm{f}, K$ is near the maximum value of unity. The experimental procedure employed was to first balance $C_{3}$ and $C_{4}$ at $100 \mathrm{cps}$. Then at $0.5 \mathrm{cps}$ a substitution measurement of the parallel capacitance of a suitable resistor is made. The quantity

$$
\begin{aligned}
C_{m o}=C_{x}-\left(C_{3}-C_{4}\right) K \frac{\Delta R_{1}}{R_{2}} & =\Delta C_{s}+C_{s}^{\prime} \frac{\Delta R_{1} K}{R_{4}} \\
& +\left(R_{3}-r R_{4}-R_{1}^{\prime \prime}\right) \frac{\Delta R_{1}}{R_{2}} L
\end{aligned}
$$

is then determined from eq (10). Next the generator-to-bridge connections are reversed and $C_{3}$ and $C_{4}$ balanced once again at $100 \mathrm{cps}$. Interchanging the generator connection interchanges the generator's contribution to $C_{3}$ and $C_{4}$. Thus, if $\left(C_{3}-C_{4}\right)$ is positive at $0.5 \mathrm{cps}$, upon interchanging it will be negative. Measuring the capacitance of the resistor once again at $0.5 \mathrm{cps}$ one may compute

$$
\begin{aligned}
C_{m i}=C_{x}+\left(C_{3}-C_{4}\right) K & \frac{\Delta R_{1}}{R_{2}}=\Delta C_{s}+C_{s}^{\prime} \frac{\Delta R_{1} K}{R_{4}} \\
& +\left(R_{3}-\frac{R_{4}}{r}-R_{1}^{\prime \prime}\right) \frac{\Delta R_{1}}{R_{2}} L,
\end{aligned}
$$

from eq (10), with $J_{1}$ and $J_{2}$ interchanged. ${ }^{15}$

Subtracting $C_{m i}$ and $C_{m o}$ we have

$$
C_{m i}-C_{m o}=2\left(C_{3}-C_{4}\right) K \frac{\Delta R_{1}}{R_{2}} .
$$

Thus, $\left(C_{3}-C_{4}\right)$ may be determined.

Measurements were made upon a nominal $10^{10}$ ohm resistor. This value was chosen as a compromise between two conflicting factors. One must attempt to minimize $R_{2}$ so as to make the correction term large, and at the same time maximize $R_{2}$ so that best sensitivity to capacitative unbalance is achieved. A compromise is achieved with a value of $R_{2}$ of $10^{7}$ ohms. At $0.5 \mathrm{cps}$, with $R_{2}=10^{7}$ ohms, the capacitance can be balanced to about $\pm 0.005 \mathrm{pf}$. If $\Delta R_{1}$ $=100 \mathrm{ohms}$ the magnitude of $\Delta R_{1} / R_{2}$ will be $10^{-5}$, which corresponds to a value of $10^{10} \mathrm{ohms}$ for $R_{x}$.

The experimental data obtained upon performing this experiment are given in table 4 . The experiment was performed twice using two values for $C_{s}^{\prime}$ (100 pf and $20 \mathrm{pf}$ ). The difference between the average values of $C_{m i}$ and $C_{m o}$ was $[0.0755-0.0725]$ $\mathrm{pf}=0.003 \mathrm{pf}$. This is within the experimental accuracy of $\pm 0.005 \mathrm{pf}$. If we take this number at its face value, we can compute from eq (46) a value of about $200 \mathrm{pf}$ for $\left(C_{3}-C_{4}\right)$. If $\left(C_{3}-C_{4}\right)$ is $200 \mathrm{pf}$

15 With $J_{1}$ and $J_{2}$ interchanged the factor $\left(R_{3}-r R_{4}-R_{1}^{\prime \prime}\right)$ in eq (10) becomes

\begin{tabular}{|c|c|c|c|c|c|c|}
\hline $\begin{array}{l}\text { Generator connection } \\
\text { Ordinary } \\
\text { Ordinary } \\
\text { Interchange } \\
\text { Interchange }\end{array}$ & $\begin{array}{r}C_{s}^{\prime}(\mathrm{pf}) \\
100.000 \\
20.000 \\
100.000 \\
20.000\end{array}$ & $\begin{array}{r}\Delta C_{s}(\mathrm{pf}) \\
-0.003 \\
+0.052 \\
.010 \\
.065\end{array}$ & $\begin{array}{r}\Delta R_{1} / R_{2} \\
74.3 / 10^{7} \\
74.5 / 10^{7} \\
74.5 / 10^{7} \\
74.5 / 10^{7}\end{array}$ & $\begin{array}{r}\mathrm{a}(\mathrm{pf}) \\
0.072 \\
.014 \\
.072 \\
.014\end{array}$ & $\begin{array}{r}\mathrm{b}(\mathrm{pf}) \\
0.005 \\
.005 \\
-0.005 \\
-0.005\end{array}$ & $\begin{array}{r}C_{m}(\mathrm{pf}) \\
0.074 \\
.071 \\
.077 \\
.074\end{array}$ \\
\hline
\end{tabular}
$\left(R_{3}-R_{4} / r-R_{1}^{\prime \prime}\right)$ which, upon evaluation, gives $\left(-71 \mathrm{ohms}-R_{1}^{\prime \prime}\right)$. The magnitude of this term depends strongly upon accurate knowledge of the values of $R_{3}, R_{4}$, and $r$.

TABLE 4. The parallel capacitance $\left(C_{m}\right)$ of a nominal $10^{10} \mathrm{ohm}$ resistor measured before and after interchanging the generator to bridge connection

$\mathrm{a}=K \Delta R_{1} C_{s}^{\prime} / R_{4} ; \mathrm{b}=\left(R_{3}-r R_{4}-R_{1}^{\prime \prime}\right) \Delta R_{1} L / R_{2}$, ordinary; $\mathrm{b}=\left(R_{3}-R_{4} / r-R_{1}^{\prime \prime}\right) \Delta R_{1} L / R_{2}$, interchange; $C_{m}=\Delta C_{s}+\mathrm{a}+\mathrm{b} ;$ and $R_{1}^{\prime \prime}=\Delta R_{1}$.

These data, obtained at $0.5 \mathrm{cps}$, together with eq (46), indicate that $\left(C_{3}-C_{4}\right)$ remains negligibly small at low frequencies. 
at $0.5 \mathrm{cps}$, with $C_{g}=\infty$, the maximum value of the correction term $\left(C_{3}-C_{4}\right) \Delta R_{1} K / R_{2}$, is obtained with $R_{2}=10^{6} \mathrm{ohms}$ and is less than $0.003 \mathrm{pf}$. This is well below the uncertainty in capacitive balance under similar conditions, which is determined from eq (34) as $\pm 0.06 \mathrm{pf}$. Therefore, $\left(C_{3}-C_{4}\right)$ remains sufficiently low that its effect upon capacitance measurements is always less than the limits of sensitivity. ${ }^{16}$

\section{Measurements upon Dielectric Materials}

As a final check upon the performance of the bridge (particularly near the high frequency end) measurements were made upon dielectric specimens and compared with results obtained using a two terminal Schering bridge (General Radio 716-C).

The two materials chosen were polymethylmethacrylate (PMMA), which has a fairly high value for the loss index $\left(\epsilon^{\prime \prime}\right)$; and a specimen of fused silica, which has a low loss index. These specimens were circular disks whose physical dimensions are given in table 5 .

TABLE 5. Dimensions of the disk shaped dielectric specimens

\begin{tabular}{|c|c|c|c|}
\hline Material & Thickness & $\begin{array}{c}\text { Area of plane } \\
\text { face }\end{array}$ & $C_{\text {o }}$ \\
\hline $\begin{array}{l}\text { PMMA } \\
\text { Fused silica }\end{array}$ & $\begin{array}{r}0.1627 \mathrm{~cm} \\
.1435 \mathrm{~cm}\end{array}$ & $\begin{array}{r}\text { 11. } 310 \mathrm{~cm}^{2} \\
7.554 \mathrm{~cm}^{2}\end{array}$ & $\begin{array}{l}\text { 6. } 155 \mathrm{pf} \\
\text { 4. } 661 \mathrm{pf}\end{array}$ \\
\hline
\end{tabular}

In order to eliminate series air films, the plane surfaces of the PMMA specimens were covered with tin-foil-petrolatum electrodes while the fused silica specimen received electrodes of evaporated gold.

A brief description of the experimental technique used in measuring $\epsilon^{\prime}$ and $\epsilon^{\prime \prime}$ follows. (A more complete description is given in ref. [7].) A micrometer holder, having plane circular electrodes 2 in. in diameter was employed. The diameters of the specimens were less than that of the electrodes in order to assure that the field within the specimen was uniform. The dielectric constant is computed from the measured parallel capacitance, $C_{x}$, according to the equation

$$
\epsilon^{\prime}=\frac{C_{x}-C_{h}}{C_{v}}+\frac{t_{s}}{t_{h}}
$$

where $t_{h}$ is the separation of the electrodes with the sample between, $t_{s}$ is the specimen thickness, and

${ }^{16} \mathrm{~A}$ mathematically simplified method of measuring $C_{3}-C_{4}$ is given here. If one replaces $C_{\mathrm{g}}$ with a resistor $R_{\mathrm{g}}$, then $\left(C_{3}-C_{4}\right)$ can be measured at low frequencies without using eq (10) which contains frequency dependent terms. If
$R_{g}=500 \mathrm{kohms}$, then eq (22) is valid if one uses a larger value of $K$. ( $R_{g}$ is inserted $R_{g}=500 \mathrm{kohms}$, then eq (22) is valid if one uses a larger value of $K$. ( $\left(R_{g}\right.$ is inserted
in order to increase $K$ and thus increase the correction term containing $\left(C_{3}-C_{4}\right)$.) in order to increase $K$ and thus increase the correction term containing $\left(C_{3}-C_{4}\right)$.)
The value of $K$ becomes $R_{m} /\left(R_{m}+R_{4}\right)$ where $R_{m}$ (the equivalent resistance The value of $K$ becomes $R_{m} /\left(R_{m}+R_{4}\right)$ where $R_{m}$ (the equivalent resistance
shunting $R \mathrm{~A})$ is $J_{2}+R_{g}\left(1+J_{2} / J_{1}\right)$. Since $J_{2}=15.8 \mathrm{kohms}, J_{2} / J_{1} \cong 1$, and $R_{g}=500$ kohms, then $R_{m}=1.016 \times 10^{6} \mathrm{ohms}$ and $K=0.91$.

The experimental procedure outlined previously is unchanged but now from eq $(22)$,

$$
C_{m o}=\Delta_{s}^{\prime} C+C_{s}^{\prime} K \frac{\Delta R_{1}}{R_{4}}=C_{x}-\left(C_{3}-C_{4}\right) K \frac{\Delta R_{1}}{R_{2}} \text {, where } K=0.91 .
$$

This expression contains no frequency dependent correction terms, thus the This expression contains no frequency dependent correction terms, thus the
analysis of the results obtained when the terminals are interchanged is simplified.
$C_{h}$ is the capacitance of the holder measured without the specimen in place and with the electrode separation equal to $t_{h}$. The loss index is obtained from eq (37).

The results of measurements made at room temperature upon the PMMA specimen are shown in figure 9 . No visible discontinuity exists in the data obtained on the two bridges. The data in the region in which the frequencies overlap is also presented in table 6. The maximum difference in $\epsilon^{\prime}$ is 0.0016 . This is a difference of only 0.05 percent. The values of $\epsilon^{\prime \prime}$ agree to within 0.2 percent.

Table 6. Comparison of dielectric constant and loss index obtained on the low frequency apparatus and on a Schering bridge for PMMA.

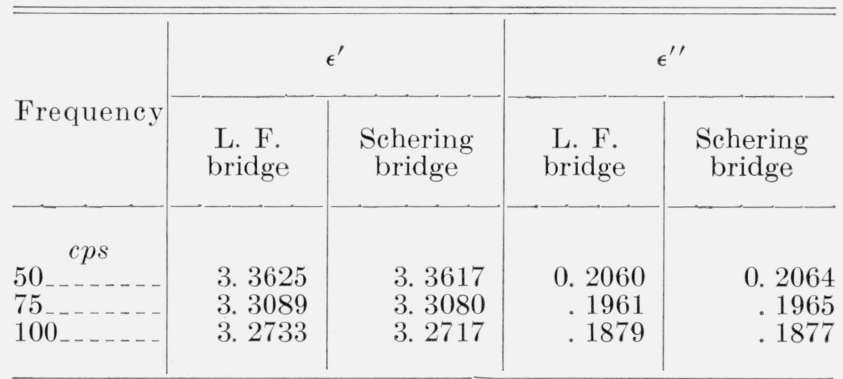

Results for the specimen of fused silica are presented in figure 10. The difference in the values of $\epsilon^{\prime}$ obtained on the two bridges is, as before, 0.05 percent. The $\epsilon^{\prime \prime}$ data of figure 10 may be joined by a smooth curve even though there is a difference of 0.00035 in the values measured at $100 \mathrm{cps}$. This difference is well within the stated accuracy of the Schering bridge, and may thus be largely associated with the scatter of the Schering bridge data. The values of $\epsilon^{\prime \prime}$ obtained on the low frequency apparatus fall within 0.0001 of the drawn curve, which is in keeping with our calculated accuracy of $\pm(1 \%+5 \times$ $\left.10^{-4} / C_{v}(\mathrm{pf})\right)$.

\section{Comments and Discussion}

It has been demonstrated (section 4.2) that the stray impedances in the generator $Z_{1}, Z_{2}$, and $Z_{L}$ produce no ill effects upon the operation of the bridge. The fact that this is true for the particular generator employed does not necessarily imply that the same is true of another generator, even though it be of the same manufacture. If another generator were employed, it would be necessary to perform those experiments which demonstrate the effects of those impedances.

It bas been shown that it is preferable to operate the bridge with $C_{\mathrm{g}}$ of figure 1 shorted. In this mode of operation, where eqs (22) and (23) apply, the correction terms are small in magnitude and essentially frequency independent.

In its present state, this apparatus can be profitably employed to investigate the dielectric properties of materials at low frequencies. The present meas- 


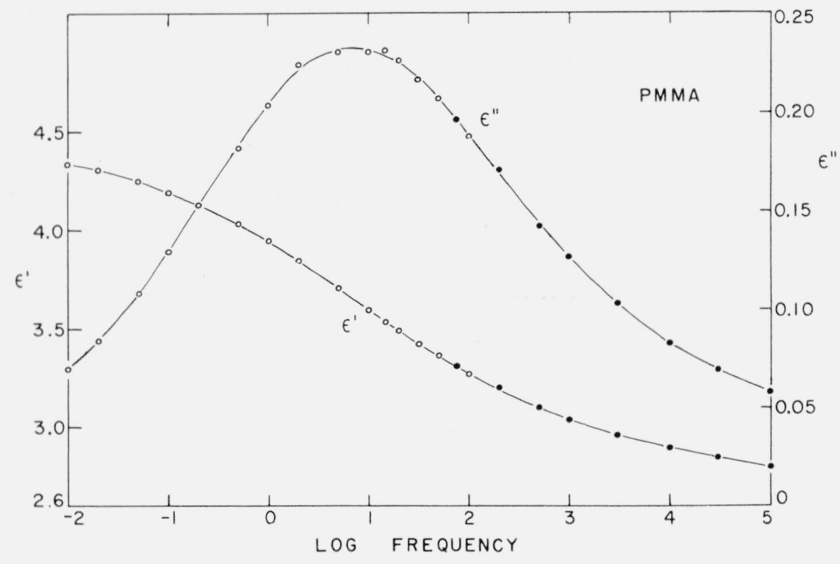

Figure 9. Dielectric constant, $\epsilon^{\prime}$, and loss index, $\epsilon^{\prime \prime}$, versus log frequency for desiccated specimen of PMMA at $23.7^{\circ} \mathrm{C}$.

-low frequency apparatus

- Schering bridge

urable range of capacitances, 0 to $100 \mathrm{pf}$, could be extended through use of larger range variable capacitors, $C_{s}$ and $C_{B}$. This alteration would make two minor changes in the behavior of the bridge. The correction terms of eqs (22) and (23), whose magnitude depends upon $C_{s}^{\prime}$, would be correspondingly increased, and the sensitivity to bridge unbalance would be decreased under certain conditions as shown in eqs (32) and (33).

The range of conductance presently measurable, $10^{-15}$ to $10^{-9}$ mhos, is adequate for most purposes. It is appropriate, however, to consider means whereby higher values of conductances may be measured. Since $G_{x}=\Delta R_{1} / R_{2} R_{4}$, higher values of conductance may be measured by decreasing $R_{2}$ and/or $R_{4}$, or by increasing $\Delta R_{1}$.

Eqs (22) and (23) remain adequate approximations if any of the above alternations are made. If $R_{4}$ is lowered to $10^{4}$ ohms, the correction term $C_{s}^{\prime} \Delta R_{1} K / R_{4}$, of eq $(22)$, would increase from a maximum of $0.019 \mathrm{pf}$ to $0.85 \mathrm{pf}$. A correction term of this magnitude is almost too large to be tolerated. Furthermore, if $R_{4}$ were $10^{4} \mathrm{ohms}$, the correction term of eq (23) would become almost doubled in magnitude. Decreasing $R_{4}$ is therefore not recommended.

If the minimum value of $R_{2}$ were made $10^{5} \mathrm{ohms}$, and $R_{4}$ remained $10^{5}$ ohms, conductances of $10^{-8}$ mhos could be measured, and the correction terms of eqs (22) and (23) would not be altered. It should be mentioned, however, that if $R_{2}$ were $10^{5}$ ohms, the capacitive sensitivity at low frequencies would suffer severely. (See eq (33) or eq (36)).

If the range of $\Delta R_{1}$ were increased to $10^{3} \mathrm{ohms}$, the following effects would result. From eq (23), it is apparent that conductances previously measurable with a particular value of $R_{2}$ would become measurable on the next higher value. Therefore, under

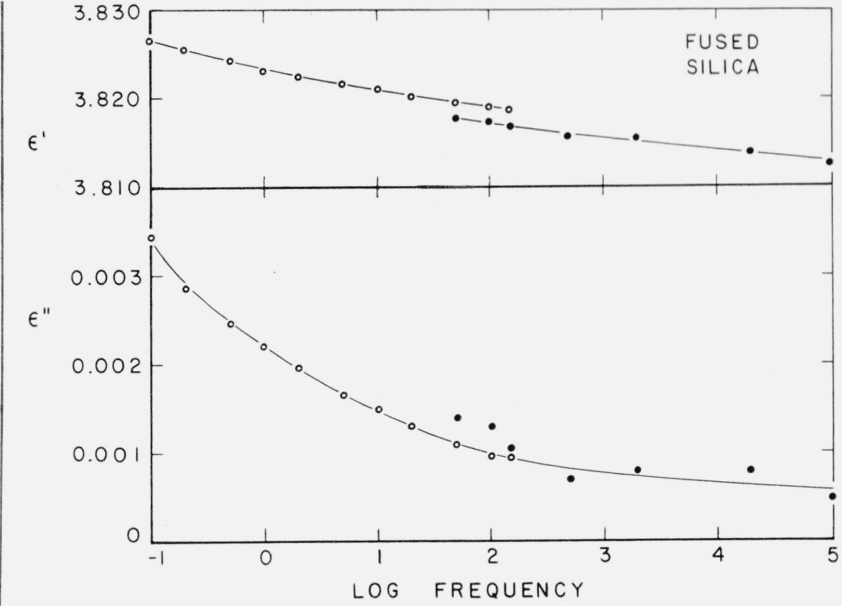

FIgure 10. Dielectric constant, $\epsilon^{\prime}$, and loss index, $\epsilon^{\prime \prime}$, versus log frequency for specimen of fused silica at $24.2^{\circ} \mathrm{C}$.

-low frequency apparatus

- - Schering bridge

The difference in the value of $\epsilon^{\prime}$ measured on the low-frequency apparatus and that obtained on the Schering bridge at $100 \mathrm{cps}$ is only $0.05 \%$

most circumstances, the sensitivity to capacitance unbalance would increase (see eq (36)). If $\Delta R_{1}$ were increased to $10^{3} \mathrm{ohms}$, however, the maximum value of the corrections in eq (22) would be increased by a factor of ten. The maximum of $C_{s}^{\prime} \Delta R_{1} K / R_{4}$ would become $0.189 \mathrm{pf}$, which is not excessively large.

Either of the last-mentioned alterations offer possibilities of increasing the range of measurable conductances without adversely affecting bridge accuracy. If used together; i.e., $R_{2}=10^{5}$ ohms, $\Delta R_{1}=10^{3}$ ohms, while $R_{4}=10^{5}$ ohms, conductances as high as $10^{-7}$ mhos could be measured.

It is informative to compare the performance of this apparatus with that of other low-frequency bridges. In table 7 the frequency range, the capacitance and conductance ranges, and the sensitivity of this apparatus is compared with that of two other bridges. Referring to table 7 , it is apparent that the apparatus studied here (a) may be operated about a decade higher in frequency, (b) has better conductance sensitivity and (c) has, in general, better capacitance sensitivity. Both the bridge of Weingarten and that of Nakajima measure capaci-

TABLE 7. Comparison of three low frequency bridges

\begin{tabular}{|c|c|c|c|c|c|}
\hline \multirow{2}{*}{ Bridge } & \multicolumn{2}{|c|}{ Conductance } & \multicolumn{2}{|c|}{ Capacitance } & \multirow{2}{*}{$\begin{array}{l}\text { Frequency } \\
\text { range }\end{array}$} \\
\hline & Range & Sensitivity a & Range & $\begin{array}{l}\text { Sensi- } \\
\text { tivity }\end{array}$ & \\
\hline $\begin{array}{l}\text { Weingarten }[2] \ldots \\
\text { Nakajima [5] } \\
\text { This apparatus.-- }\end{array}$ & $\begin{array}{c}(\text { mho }) \\
10^{-9} \text { to } 10^{-14} \\
10^{-7} \text { to } 10^{-13} \\
10^{-9} \text { to } 10^{-15}\end{array}$ & $\begin{array}{l}(m h o) \\
\sim 5 \times 10^{-15} \\
\sim 10^{-13} \\
\sim 3 \times 10^{-16}\end{array}$ & $\begin{array}{l}(p f) \\
\text { to } 1000 \\
\text { to } 800 \\
\text { to } 100\end{array}$ & $\begin{aligned} & (p f) \\
\sim & 0.1 \\
\sim & 0.2 \\
\sim & 0.001\end{aligned}$ & $\begin{array}{c}(c p s) \\
10 \text { to } 0.008 \\
10 \text { to } 0.008 \\
200 \text { to } 0.008\end{array}$ \\
\hline
\end{tabular}

a The sensitivities given here are valid for frequencies near $0.1 \mathrm{cps}$. A detailed discussion of the sensitivity of this apparatus is given in section 3.5. 
tances near 1000 pf. The present bridge may be modified to do so also. The bridge of Nakajima can measure conductances as high as $10^{-7}$ mhos. Alterations of the present bridge, whereby conductances of this magnitude can be measured, have previously been discussed.

The present bridge has been subjected to a detailed theoretical and experimental analysis, which gives confidence, within the limits stated, in the absolute values of the dielectric properties obtained with it. It is well suited to the investigation of dielectric relaxation phenomena in materials where the loss is low, and where molecular mechanisms lead to long relaxation times.

\section{Appendix}

\subsection{Derivation of the Bridge Balance Equation ${ }^{17}$}

Referring to figure 2(b) we see that the Y network consisting of $J_{1}, Z_{g}, J_{2}$ may be transformed into a $\Delta$ network containing an impedance across $Z_{3}$ which we shall call $Z_{n}$ and an impedance across $Z_{A}$ which we shall call $Z_{m}$.

The transformation equations are

$$
\begin{aligned}
& Z_{n}=J_{1}+Z_{g}\left(1+\frac{J_{1}}{J_{2}}\right) \\
& Z_{m}=J_{2}+Z_{g}\left(1+\frac{J_{2}}{J_{1}}\right) .
\end{aligned}
$$

The balance equations of the resulting Wheatstone network can be written as

$$
\left[\frac{Z_{3} Z_{n}}{Z_{3}+Z_{n}}\right] Z_{B}=\left[\frac{Z_{A} Z_{m}}{Z_{A}+Z_{m}}\right] Z_{P}
$$

Setting $J_{1} / J_{2}=r$, substituting for $Z_{n}$ and $Z_{m}$ in this expression one obtains the balance condition stated in eq (2), i.e.

$$
\frac{1}{Z_{P}}\left[r+\frac{Z_{g}}{Z_{A}}(1+r)+\frac{J_{1}}{Z_{A}}\right]=\frac{1}{Z_{B}}\left[1+\frac{Z_{g}}{Z_{3}}(1+r)+\frac{J_{1}}{Z_{3}}\right] .
$$

\subsection{Exact Solutions of the General Bridge Equations}

The exact solutions of the bridge balance equations with $C_{g}$ in the circuit are obtained by combining eqs (6), (7), (8), and (9) and solving for $C_{x}$ and $1 / R_{x}$. The solution for $C_{x}$ is

$$
C_{x}=C_{s}^{\prime}-C_{s}^{\prime \prime}+A+B+C+E
$$

17 The author wishes to thank F. K. Harris for suggesting this derivation of eq $(\mathrm{A}-3)$. where

$$
\begin{aligned}
A= & \frac{C_{s}^{\prime}}{D}\left(\frac{1}{R_{A}^{\prime}}-\frac{1}{R_{A}^{\prime \prime}}\right) R_{A}^{\prime \prime} \\
& {\left[1+\frac{R_{A}^{\prime \prime}}{R_{B}^{\prime}}+\frac{\omega^{2} R_{A}^{\prime \prime} J_{1} C_{g}^{2}}{(1+r)^{2}}\left(r+\frac{J_{1}}{R_{A}^{\prime \prime}}+\frac{J_{1}}{R_{B}^{\prime}}\right)\right] } \\
& -\frac{C_{B}}{D}\left(\frac{1}{R_{B}^{\prime \prime}}-\frac{1}{R_{B}^{\prime}}\right) R_{A}^{\prime \prime}\left[\frac{R_{A}^{\prime \prime}}{R_{3}}+\frac{\omega^{2} R_{A}^{\prime \prime} J_{1} C_{g}^{2}}{(1+r)^{2}}\left(1+\frac{J_{1}}{R_{3}}\right)\right], \\
B= & \frac{C_{g}}{D}\left(\frac{1}{R_{B}^{\prime \prime}}-\frac{1}{R_{B}^{\prime}}\right) \frac{R_{A}^{\prime \prime}}{(1+r)}\left[1+\frac{R_{A}^{\prime \prime}}{R_{B}^{\prime}}-\frac{r R_{A}^{\prime \prime}}{R_{3}}\right], \\
C= & \frac{C_{3}}{D}\left(\frac{1}{R_{B}^{\prime \prime}}-\frac{1}{R_{B}^{\prime}}\right) R_{A}^{\prime \prime} \\
& -\frac{C_{4}}{D}\left(\frac{1}{R_{B}^{\prime \prime}}-\frac{1}{R_{B}^{\prime}}\right) R_{A}^{\prime \prime}\left[\frac{R_{A}^{\prime \prime}}{R_{3}}+\frac{\omega^{2} R_{A}^{\prime \prime} J_{1} C_{g}^{2}}{(1+r)^{2}}\left(1+\frac{J_{1}}{R_{3}}\right)\right],
\end{aligned}
$$

and

$$
\begin{aligned}
E=\frac{1}{D R_{s}}\left(\frac{1}{R_{A}^{\prime \prime}}-\frac{1}{R_{A}^{\prime}}\right) \frac{C_{g} R_{A}^{\prime \prime 2}}{(1+r)} & \\
& {\left[r+\frac{(1+r)\left(C_{4}+C_{B}\right)}{C_{g}}+\frac{\omega^{2} J_{1}^{2} C_{g}\left(C_{4}+C_{B}\right)}{(1+r)}\right] . }
\end{aligned}
$$

The expression for the conductance is

$$
G_{x}=\frac{1}{R_{x}}=\frac{1}{R_{B}^{\prime \prime}}-\frac{1}{R_{B}^{\prime}}+M+N+O+P+Q
$$

where

$$
\begin{aligned}
& M=\frac{1}{D}\left[C_{\mathrm{s}}^{\prime}\left(\frac{1}{R_{A}^{\prime}}-\frac{1}{R_{A}^{\prime \prime}}\right)-C_{B}\left(\frac{1}{R_{B}^{\prime \prime}}-\frac{1}{R_{B}^{\prime}}\right)\right] \\
& {\left[\omega^{2} R_{A}^{\prime \prime 2} C_{g}\left(\frac{C_{4}}{C_{g}}+\frac{r}{(1+r)}\right)+\frac{\omega^{4} \tau_{1}^{4}}{\left(C_{4}+C_{B}\right)}\right],} \\
& N=\frac{\left(C_{3}-C_{4}\right)}{D}\left(\frac{1}{R_{B}^{\prime \prime}}-\frac{1}{R_{B}^{\prime}}\right) \\
& {\left[\omega^{2} R_{A}^{\prime \prime 2} C_{g}\left(\frac{C_{4}}{C_{g}}+\frac{r}{(1+r)}\right)+\frac{\omega^{4} \tau_{1}^{4}}{\left(C_{4}+C_{B}\right)}\right]} \\
& O=\frac{1}{D}\left(\frac{R_{A}^{\prime \prime}}{R_{3}}-1-\frac{R_{A}^{\prime \prime}}{R_{B}^{\prime}}\right)\left(\frac{1}{R_{B}^{\prime \prime}}-\frac{1}{R_{B}^{\prime}}\right) \\
& {\left[1+\frac{\omega^{2} J_{1} R_{A}^{\prime \prime} C_{g}^{2}\left(r+J_{1} / R_{A}^{\prime \prime}\right)}{(1+r)^{2}}\right]} \\
& P=\frac{(1-r)}{D}\left(\frac{1}{R_{B}^{\prime \prime}}-\frac{1}{R_{B}^{\prime}}\right) \frac{\omega^{2} R_{A}^{\prime \prime 2} C_{g}^{2}}{(1+r)^{2}}\left[\frac{J_{1}}{R_{A}^{\prime \prime}}+r+(1+r) \frac{C_{4}}{C_{g}}\right] \text {, }
\end{aligned}
$$


and

$$
Q=\frac{R_{A}^{\prime \prime}}{D R_{s}}\left(\frac{1}{R_{A}^{\prime}}-\frac{1}{R_{A}^{\prime \prime}}\right)\left[1+\frac{\omega^{2} J_{1} R_{A}^{\prime \prime} C_{g}^{2}}{(1+r)^{2}}\left(r+J_{1} / R_{A}^{\prime \prime}\right)\right] .
$$

In eqs (A-4) and (A-5), $D$ is given by

$$
D=1+R_{A}^{\prime \prime} / R_{B}^{\prime}+\omega^{2} \tau_{0}^{2}+\omega^{4} \tau_{1}^{4},
$$

where

$$
\begin{aligned}
\tau_{0}^{2}=\frac{C_{g}^{2} R_{A}^{\prime \prime 2}}{(1+r)^{2}} & {\left[\left(r+\frac{J_{1}}{R_{A}^{\prime \prime}}+(1+r) \frac{C_{4}}{C_{g}}\right)^{2}+\frac{J_{1}}{R_{B}^{\prime}}\left(r+\frac{J_{1}}{R_{A}^{\prime \prime}}\right)\right.} \\
& \left.+\frac{(1+r) C_{B}}{C_{g}}\left(r+(1+r) \frac{C_{4}}{C_{g}}\right)-\frac{2 J_{1} C_{4}(1+r)}{R_{A}^{\prime \prime} C_{g}}\right]
\end{aligned}
$$

and

$$
\tau_{1}^{4}=C_{g}^{2} C_{4}\left(C_{4}+C_{B}\right) R_{A}^{\prime \prime 2} J_{1}^{2} /(1+r)^{2} .
$$

Approximate solutions with $\mathrm{C}_{g}=1 \mu \mathrm{f}$. Let us now evaluate the various terms of eqs $(\mathrm{A}-4)$ and $(\mathrm{A}-5)$ and drop those which are clearly unimportant. We shall deal first with the case wherein $C_{g}$ is $1 \mu \mathrm{f}$ and begin by evaluating $\omega^{2} \tau_{0}^{2}$ and $\omega^{4} \tau_{1}^{4}$. In order to do so we must obtain the value of $C_{4}$. In appendix 7.4, $C_{4}$ is determined to be $1450 \mathrm{pf} \pm 10$ percent. Additional leads may be employed in the future, thus increasing $C_{4}$. It will however, be assumed that $C_{4} \leqq 2000$ pf. Using the values for the constant parameters given in table 1 , and the value of 0.99859 for $r$, (determined in section 4.2 a), we obtain $\omega^{4} \tau_{1}^{4} \leq$ $3.89 \times 10^{-9} f^{4}$. Since $R_{B}^{\prime} \geq 10^{9}$ ohms, $R_{A}^{\prime \prime} \simeq R_{4} \cong 10^{5}$ ohms, and $C_{B} \leq 125 \mathrm{pf}$, the expression for $\omega^{2} \tau_{0}^{2}$, to an accuracy of about 0.8 percent, becomes

$$
\omega^{2} \tau_{0}^{2}=\frac{\omega^{2} C_{g}^{4} J_{2}^{2}}{(1+r)^{2}}\left(1+\frac{R_{4}}{J_{2}}\right)^{2} .
$$

Upon evaluation this becomes: $\omega^{2} \tau_{0}^{2}=0.132 f^{2}$. At frequencies less than about 600 cps $\omega^{2} \tau_{0}^{2} \geq 100$ $\left(\omega^{4} \tau_{1}^{4}\right)$. Since the bridge is operated with $f<600$ cps, then $\omega^{4} \tau_{1}^{4}$ is negligible. To an accuracy of 0.8 percent or better, $D$ is given by

$$
D=1+\omega^{2} \tau_{0}^{2}
$$

In order to evaluate the terms in eqs $(\mathrm{A}-4)$ and $(\mathrm{A}-5)$ the following identities are used

$$
\begin{gathered}
\frac{1}{R_{A}^{\prime}}-\frac{1}{R_{A}^{\prime \prime}}=\left(1+\frac{R_{4}}{R_{2}}\right) \frac{\Delta R_{1}}{R_{A}^{\prime} R_{A}^{\prime \prime}} \\
\frac{1}{R_{B}^{\prime \prime}}-\frac{1}{R_{B}^{\prime \prime}}=\frac{R_{4}}{R_{2}} \frac{\Delta R_{1}}{R_{A}^{\prime} R_{A}^{\prime \prime}} .
\end{gathered}
$$

These relationships are derived from eq (1) of section 3.2 .

Since $R_{s} \geq 10^{11}$ ohms the quantity $E$ in eq $(\mathrm{A}-4)$ contributes less than 0.001 pf and may therefore be omitted. The expressions for $A, B$, and $C$, which may contribute terms greater than $0.001 \mathrm{pf}$, may be reduced to the following with the aid of eqs $(\mathrm{A}-7)$ $(\mathrm{A}-8)$, and $(\mathrm{A}-9)$

and

$$
\begin{array}{r}
A^{\prime}=C_{s}^{\prime} \frac{\Delta R_{1}\left(1+\omega^{2} \tau_{0}^{2}+R_{4} / J_{2}\right)}{R_{4}\left(1+\omega^{2} \tau_{0}^{2}\right)\left(1+R_{4} / J_{2}\right)}, \\
B^{\prime}=\frac{C_{g} \Delta R_{1}\left(R_{3}-r R_{4}-R_{1}^{\prime \prime}\right)}{2 R_{4} R_{2}\left(1+\omega^{2} \tau_{0}^{2}\right)},
\end{array}
$$

$$
C^{\prime}=\left(C_{3}-C_{4}\right) \frac{\Delta R_{1}\left(1+\omega^{2} \tau_{0}^{2}+R_{4} / J_{2}\right)}{R_{2}\left(1+\omega^{2} \tau_{0}^{2}\right)\left(1+R_{4} / J_{2}\right)} .
$$

In eqs $(\mathrm{A}-10)$ use is made of the approximations

$$
\begin{gathered}
R_{A}^{\prime \prime} \cong R_{A}^{\prime} \cong R_{4} \cong R_{3}, \\
J_{1} \simeq J_{2},
\end{gathered}
$$

and

$$
r C_{g} \gg(1+r) C_{4}
$$

The approximations $(\mathbf{A}-11)$ are accurate to about 0.1 percent. These approximations are not applied however in simplifying the expression $\left(1+R_{A}^{\prime \prime} / R_{B}^{\prime}-\right.$ $\left.r R_{A}^{\prime \prime} / R_{3}\right)$. From eq (1) this becomes $\left(R_{3}-r R_{4}-\right.$ $\left.R_{1}^{\prime \prime}\right) / R_{3}$ to an accuracy of about $\pm 10 \mathrm{ohms} / R_{3}$.

The expression for $C_{x}$ becomes

$$
C_{x}=C_{s}^{\prime}-C_{s}^{\prime \prime}+A^{\prime}+B^{\prime}+C^{\prime} .
$$

Further evaluation of $A^{\prime}, B^{\prime}$, and $C^{\prime}$ is given in section 3.3 .

In the equation for $G_{x}$, eq $(\mathrm{A}-5)$, it can be shown that the terms $O$ and $P$ will constitute less than 0.2 percent of $G_{x}$, and may be omitted since $\left|R_{A}^{\prime \prime}\right| R_{3}-1 \mid$ $<0.001$ and $(1-r)<0.002$. Also since $\left(C_{3}-C_{4}\right)$ $<600$ pf (see section 3.2a), $N$ is less than 0.2 percent of $G_{x}$ and may be omitted if $f \leq 200 \mathrm{cps}$. $Q$ may be omitted to an accuracy of at least 0.1 percent since $R_{s} \geq 10^{3} R_{2}$. $M$ can become significant and is retained. Using eqs $(\mathrm{A}-7),(\mathrm{A}-8),(\mathrm{A}-9)$, and $(\mathrm{A}-11)$ the expression for the conductance may be written

$$
G_{x}=\frac{\Delta R_{1}}{R_{2} R_{4}}\left[1+M^{\prime}\right]
$$

where

$$
M^{\prime}=\frac{C_{s}^{\prime} R_{2}}{R_{4} C_{g}\left(1+J_{1} / R_{4}\right)^{2}}\left[\frac{\omega^{2} \tau_{0}^{2}}{1+\omega^{2} \tau_{0}^{2}}\right]\left(2+\omega^{2} C_{4} C_{g} J_{1}^{2}\right) .
$$

This expression for $M^{\prime}$ is accurate to at least 0.8 percent. Further discussion of eq $(\mathrm{A}-13)$ is given in section 3.3 .

\subsection{Exact Solutions of Bridge Equations with $\mathrm{C}_{g}=\infty$}

The bridge is ordinarily operated with $C_{g}=\infty$. The exact solutions, eqs $(\mathrm{A}-4)$ and $(\mathrm{A}-5)$, of the bridge balance conditions are simplified with $C_{\mathrm{g}}=\infty$. The solution for $C_{x}$ is

$$
C_{x}=C_{s}^{\prime}-C_{s}^{\prime \prime}+a+c+e
$$


where

$$
\begin{aligned}
a=\frac{J_{1}}{d}\left[C_{s}^{\prime}\left(r+\frac{J_{1}}{R_{A}^{\prime \prime}}+\frac{J_{1}}{R_{B}^{\prime}}\right)\right. & \left(\frac{1}{R_{A}^{\prime}}-\frac{1}{R_{A}^{\prime \prime}}\right) \\
& \left.-C_{B}\left(1+\frac{J_{1}}{R_{3}}\right)\left(\frac{1}{R_{B}^{\prime \prime}}-\frac{1}{R_{B}^{\prime}}\right)\right],
\end{aligned}
$$

$c=\frac{J_{1}}{d}\left(\frac{1}{R_{B}^{\prime \prime}}-\frac{1}{R_{B}^{\prime}}\right)\left[C_{3}\left(r+\frac{J_{1}}{R_{A}^{\prime \prime}}+\frac{J_{1}}{R_{B}^{\prime}}\right)-C_{4}\left(1+\frac{J_{1}}{R_{3}}\right)\right]$

$e=\frac{\left(C_{B}+C_{4}\right)}{d} \frac{J_{1}^{2}}{R_{s}}\left(\frac{1}{R_{A}^{\prime \prime}}-\frac{1}{R_{A}^{\prime}}\right)$.

The conductance is given by

$$
G_{x}=\frac{1}{R_{B}^{\prime \prime}}-\frac{1}{R_{B}^{\prime}}+m+n+p+q
$$

where

$$
\begin{aligned}
& m=\left[C_{s}^{\prime}\left(\frac{1}{R_{A}^{\prime}}-\frac{1}{R_{A}^{\prime \prime}}\right)-C_{B}\left(\frac{1}{R_{B}^{\prime \prime}}-\frac{1}{R_{B}^{\prime}}\right)\right] \frac{\omega^{2} J_{1}^{2} C_{4}}{d}, \\
& n=\frac{\left(C_{3}-C_{4}\right)}{d}\left(\frac{1}{R_{B}^{\prime \prime}}-\frac{1}{R_{B}^{\prime}}\right) \omega^{2} J_{1}^{2} C_{4}, \\
& p=\left[(1-r)+\frac{J_{1}}{R_{2}}\left(1-\frac{R_{3}}{R_{A}^{\prime \prime}}\right)-\frac{J_{1}}{R_{B}^{\prime}}\right]\left(\frac{1}{R_{B}^{\prime \prime}}-\frac{1}{R_{B}^{\prime}}\right) \frac{\left(r+J_{1} / R_{A}^{\prime \prime}\right)}{d} \\
& q=\frac{J_{1}}{R_{s} d}\left(r+\frac{J_{1}}{R_{A}^{\prime \prime}}\right)\left(\frac{1}{R_{A}^{\prime}}-\frac{1}{R_{A}^{\prime \prime}}\right),
\end{aligned}
$$

and

$$
d=\left(r+\frac{J_{1}}{R_{A}^{\prime \prime}}\right)^{2}+\frac{J_{1}}{R_{B}^{\prime}}\left(r+\frac{J_{1}}{R_{A}^{\prime \prime}}\right)+\omega^{2} J_{1}^{2} C_{4}\left(C_{4}+C_{B}\right) \cdot{ }^{18}
$$

Approximate solution with $C_{g}=\infty$. With the aid of eq (1) and the values given in table 1 , the factors in the expression for $d$ can be evaluated. Noting that $J_{1} / R_{B}^{\prime}<2 \times 10^{-5}$, and that $\omega^{2} J_{1}^{2} C_{4}\left(C_{4}+C_{B}\right)<2 \times$ $10^{-3}$ when $\mathrm{f} \leq 200 \mathrm{cps}, d$ becomes,

$$
d=\left(r+J_{1} / R_{A}^{\prime \prime}\right)^{2},
$$

to an accuracy of at least \pm 0.2 percent.

In eq $(\mathrm{A}-15)$, since $R_{s} \geq 10^{7} \mathrm{ohms}$, the term $e$ is less than 0.001 pf and may be omitted. The terms $a$ and $c$, which may be greater than $0.001 \mathrm{pf}$, are simplified using eqs $(\mathrm{A}-9),(\mathrm{A}-17)$, and $(\mathrm{A}-11)$, and become

\footnotetext{
18 The exact solutions of the bridge equations given by eqs $(A-4)$, and $(A-5)$ and by $(\mathrm{A}-15)$ and $(\mathrm{A}-16)$ are expressed in terms of the equivalent resistances of arms $\mathrm{A}$ and $\mathrm{B}$, i.e., in terms of $R_{A}$ and $R_{B}$. For the case where $R_{1}$ is a variable resistor, eqs (1) and (A-9) are used to evaluate terms containing $R_{A}$ and $R_{B}$. However, if a different resistive device were employed to balance the conductance, eqs $(\mathrm{A}-4)$ and $(\mathrm{A}-5)$ or eqs $(\mathrm{A}-15)$ and $(\mathrm{A}-16)$ may still be employed. In particular, if $R_{1}$ were a resistive voltage divider whose variable contact was connected to $R_{2}$, these general equations would still apply. Equations similar to eqs (1) and (A-9) can be derived for the case wherein $R_{1}$ is a resistive voltage divider, and with these, the exact equations simplified.

The proposed use of a resistive voltage divider in place of a variable $R_{1}$ would tend to hold the total resistance of $R_{A}$ more nearly constant thus reducing the magnitude of correction terms arising from changes in $R_{1}$.
}

$$
\begin{aligned}
& a^{\prime}=\frac{C_{s}^{\prime} \Delta R_{1}}{R_{4}\left(1+R_{4} / J_{2}\right)}, \\
& c^{\prime}=\frac{\left(C_{3}-C_{4}\right) \Delta R_{1}}{R_{2}\left(1+R_{4} / J_{2}\right)} .
\end{aligned}
$$

Our expression for $C_{x}$ becomes

$$
C_{x}=C_{s}^{\prime}-C_{s}^{\prime \prime}+a^{\prime}+c^{\prime} .
$$

Further discussion of eq $(\mathbf{A}-19)$ is given in section 3.4 .

In the expression for $G_{x}(\mathrm{eq}(\mathrm{A}-16))$, the term $p$ may be dropped since $\left|R_{3} / R_{A}-1\right|<0.001$ and $(1-\mathrm{r})<0.002$. Also, $n$ may be omitted to an accuracy of 0.1 percent or better, since $\mathrm{f} \leq 200 \mathrm{cps}$ and $\left(C_{3}-C_{4}\right)<2500 \mathrm{pf}$. The term $q$ may be dropped since it is less than 0.1 percent when $R_{s}>R_{2} / 100$. The quantity $m$, however, is not always negligible and must be retained.

Using eqs $(\mathrm{A}-9),(\mathrm{A}-17)$, and $(\mathrm{A}-11)$ the expression for $G_{x}$ may be written:

$$
G_{x}=\frac{\Delta R_{1}}{R_{4} R_{2}}\left[1+m^{\prime}\right]
$$

where

$$
m^{\prime}=\frac{C_{s}^{\prime} R_{2} \omega^{2} R_{4} C_{4}}{\left(1+R_{4} / J_{2}\right)^{2}}
$$

Detailed consideration is given eq $(\mathrm{A}-20)$ in section 3.4 .

\subsection{Measurement of $\mathrm{C}_{4}$}

The total capacitance, $C_{4}$, which shunts $R_{A}$, is best measured at $100 \mathrm{cps}$ where the effects of a capacitive shunt will be most pronounced. To further enhance the relative importance of $C_{4}$, the effective value of the resistive ratio arm should be maximized. At 100 cps with either $C_{g}=\infty$ or $C_{g}=1 \mu \mathrm{f}, R_{3}$ and $R_{A}$ are effectively shunted by $J_{1}$ and $J_{2}$. If $C_{\mathrm{g}}=0$ however, no resistive shunting of $R_{3}$ and $R_{A}$ occurs, so that the equivalent resistance is maximized. Accordingly $C_{4}$ is best measured at $100 \mathrm{cps}$ with $C_{\mathrm{g}}=0$.

The method by which $C_{4}$ can be measured is developed from eqs (6) and (7). Setting $C_{g}=0$, these equations become

$$
C_{P}=C_{B} \frac{R_{A}}{R_{3}}+C_{3} \frac{R_{A}}{R_{B}}-C_{4} \frac{R_{A}}{R_{P}}
$$

and

$$
\frac{1}{R_{P}}=\frac{R_{A}}{R_{B} R_{3}}+\omega^{2} R_{A}\left(C_{P} C_{4}-C_{B} C_{3}\right)
$$

Substituting for $1 / R_{P}$, eq $(\mathrm{A}-22)$ becomes:

$$
\begin{aligned}
C_{P}=C_{B} \frac{R_{A}}{R_{3}}+\frac{R_{A}}{R_{B}}\left[C_{3}-C_{4} \frac{R_{A}}{R_{3}}\right] \\
\quad-\omega^{2} C_{4} R_{A}^{2}\left(C_{P} C_{4}-C_{B} C_{3}\right) .
\end{aligned}
$$


If $R_{1}$ is set to zero, (so that according to eq (1), $R_{B}=\infty$ ), and the bridge is balanced by varying $C_{s}$ and $C_{3}$, with no unknown in the circuit, eqs $(\mathrm{A}-22)$ and $(\mathrm{A}-23)$ give:

and

$$
C_{s}^{\prime}=C_{B} R_{4} / R_{3},
$$

or

$$
C_{s}^{\prime} C_{4}=C_{B} C_{3}^{\prime} \text {, }
$$

$$
C_{4}=\frac{C_{B}}{C_{s}^{\prime}} C_{3}^{\prime}=\frac{R_{3}}{R_{4}} C_{3}^{\prime}
$$

Here we have assumed the parallel resistance of the capacitors $C_{B}$ and $C_{s}$ to be infinite. The values of $C_{s}$ and of $C_{3}$ at balance are denoted as $C_{s}^{\prime}$ and $C_{3}^{\prime}$ respectively.

If $C_{3}$ is now increased from $C_{3}^{\prime}$ by an amount $\Delta C_{3}$. the bridge may be rebalanced by adjusting $R_{1}$ and $C_{s}$. Equation (A-24) becomes

$$
\begin{aligned}
C_{s}^{\prime}=C_{B} \frac{R_{A}^{\prime \prime}}{R_{3}} & +\frac{R_{A}^{\prime \prime}}{R_{B}^{\prime \prime}}\left[C_{3}^{\prime}+\Delta C_{3}-C_{4} \frac{R_{A}^{\prime \prime}}{R_{3}}\right] \\
& -\omega^{2} C_{4} R_{A}^{\prime \prime 2}\left[C_{s}^{\prime \prime} C_{4}-C_{B}\left(C_{3}^{\prime}+\Delta C_{3}\right)\right],
\end{aligned}
$$

where

$$
R_{A}^{\prime \prime} \cong R_{4}+R_{1}^{\prime \prime}
$$

and

$$
R_{B}^{\prime \prime} \cong \frac{R_{2} R_{4}}{R_{1}^{\prime \prime}}
$$

Here $C_{s}^{\prime \prime}$ and $R_{1}^{\prime \prime}$ represent the values of $C_{s}$ and $R_{1}$ at balance after increase of $C_{3}$.

With the help of eqs $(\mathrm{A}-22)$ we may write

$$
\begin{aligned}
& {\left[C_{3}^{\prime}+\Delta C_{3}-C_{4} \frac{R_{A}^{\prime \prime}}{R_{3}}\right]=\Delta C_{3}-C_{4} \frac{R_{1}^{\prime \prime}}{R_{3}}} \\
& {\left[C_{s}^{\prime \prime} C_{4}-C_{B}\left(C_{3}^{\prime}+\Delta C_{3}\right)\right]=C_{4} \Delta C_{s}-C_{B} \Delta C_{3},}
\end{aligned}
$$

where

$$
\Delta C_{s}=C_{s}^{\prime \prime}-C_{s}^{\prime}
$$

Substituting these quantities into eq (A-26) and subtracting $(\mathrm{A}-25)$ yields this solution for $\mathrm{C}_{4}$

$C_{4}=\frac{C_{B} \Delta C_{3}}{2 \Delta C_{s}}-\frac{R_{1}^{\prime \prime 2}}{2 R_{2} R_{3} R_{4}^{2} \omega^{2} \Delta C_{s}}$

$$
\begin{aligned}
& \pm \frac{1}{2}\left\{\left[\frac{R_{1}^{\prime \prime 2}}{R_{2} R_{3} R_{4}^{2} \omega^{2} \Delta C_{s}}-\frac{C_{B} \Delta C_{3}}{\Delta C_{s}}\right]^{2}\right. \\
& \left.-\frac{4}{\omega^{2} R_{4}^{2}}\left[1-\frac{C_{B} R_{1}^{\prime \prime}}{\Delta C_{s} R_{3}}-\frac{\Delta C_{3}}{\Delta C_{s}} \frac{R_{1}^{\prime \prime}}{R_{2}}\right]\right\}^{1 / 2}
\end{aligned}
$$

The experiment outlined above was performed at $100 \mathrm{cps}$, with $C_{B}=100 \mathrm{pf}$ and $R_{2}=10^{6}$ ohms. After the initial balance, $C_{3}$ was increased by $100 \mathrm{pf}$. The balance was restored when $\Delta C_{s}=0.099 \mathrm{pf}$ and $R_{1}$ was set to $38.8 \mathrm{ohms}$. Using these results, and the minus sign before the square root, the solution of eq $(\mathrm{A}-28)$ is

$$
C_{4}=1450 \mathrm{pf} .
$$

In order to test the accuracy of the method the experiment was repeated after adding $1000 \mathrm{pf}$ to $C_{4}$. The result obtained for $\left(C_{4}+1000 \mathrm{pf}\right)$ was $2310 \mathrm{pf}$. Comparison of these values shows that the result for $C_{4}$ is accurate to about 10 percent. This accuracy is adequate for setting an upper limit on $C_{4}$.

The value of $C_{4}$ employed is

$$
C_{4}=1450 \mathrm{pf} \pm 10 \% \text {. }
$$

\subsection{Bridge Circuit with Wagner Ground}

If one desires to operate the bridge in such a manner that the generator's impedances to ground do not affect the balance conditions then a Wagner earthing device may be used as shown in figure 11 . In this case the impedances $J_{1}, J_{2}$, and $C_{g}$ (see fig. 1) have been removed from the generator. Stray impedances to ground which may exist in the generator are now in shunt with the arms of the Wagner ground consisting of $R_{7}, R_{8}$, and $C_{7}$, where their effects may be balanced out.

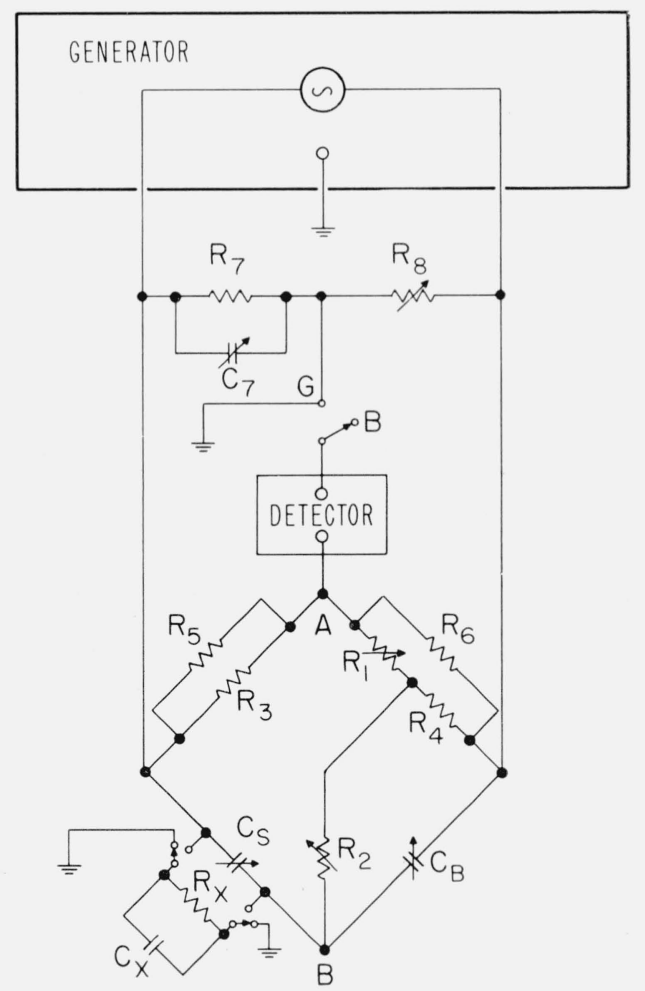

Figure 11. Proposed circuit which includes a Wagner ground. $C_{x}$ and $R_{x}$ are the unknown impedances; $C_{S}$ and $C_{B}$ variable capacitors; $R_{3}=R_{4}=10^{5}$ ohms; $R_{5}=R_{6}=R_{7}=R_{8}=20$ kohm $\left(R_{8}\right.$ variable): $R_{1}$, variable from 0 to 111 in $0.1 \mathrm{ohm}$ steps; $R_{2}$ intervariable), $R_{1}$, variable from $R^{5}$ to $R_{1}$ in 0.1 ohm steps; $R_{2}$ inter changeable $10^{5}$ to $10^{10} \mathrm{ohms}$; $C_{7}$, variable capacitor, 100 to $1000 \mathrm{pf}$. to ground which may exist within the generator. 
The circuit is balanced as follows: with the detector in position $\mathrm{G}, R_{8}$ and $C_{7}$ are varied to achieve a null. With the detector in position $\mathrm{B}, C_{s}$ and $R_{1}$ are varied to attain a null. The change in $R_{1}$ will slightly affect the original balance so that balance with the detector at $\mathrm{G}$ will have to be reestablished, etc. The circuit is balanced when a null is obtained sumultaneously at points $\mathrm{G}$ and B. By repeating this balancing procedure with the unknown "out" and then "in", $C_{x}$ and $R_{x}$ may be determined in terms of the impedances $C_{s}, R_{1}, R_{2}, R_{3}, R_{4}, R_{5}$, and $R_{6}$. The solutions for the unknowns become to a very good approximation;

and

$$
C_{x}=C_{s}^{\prime}-C_{s}^{\prime \prime}+\frac{C_{s}^{\prime}\left(R_{1}^{\prime \prime}-R_{1}^{\prime}\right) R_{6}}{R_{4}\left(R_{6}+R_{4}\right)}
$$

$$
G_{x}=\frac{R_{1}^{\prime \prime}-R_{1}^{\prime}}{R_{2} R_{4}}
$$

Here it is assumed that the stray capacitances across $R_{5}$ and $R_{6}$ are less than $10 \mathrm{pf}$.

$R_{5}$ and $R_{6}$ are placed in shunt with $R_{3}$ and $\left(R_{1}+R_{4}\right)$ respectively. Being lower in value they effectively determine the magnitude of the equivalent resistive ratio arms. Their purpose is to reduce the variation in the total value of the resistance of the arm which results from changes in $R_{1}$.

Twenty kilohms was selected as the magnitude of $R_{5}, R_{6}, R_{7}$, and $R_{8}$ so that the load on the generator would be comparable to that shown in figure 1 . Perhaps lower values of resistance could be employed without overloading the generator but this point has not been investigated.
The sensitivity of this apparatus will be formally identical with that described previously by eqs (32) and (33) as long as coupling between points $\mathrm{A}$ and ground, and B and ground is negligible.

The author is grateful to F. K. Harris, F. R. Kotter, J. D. Hoffman, and William P. Harris for their constructive reviews of the manuscript. John I. Lauritzen, Jr., is given special acknowledgement for his participation in many helpful discussions.

\section{References}

[1] J. Volger, J. M. Stevels, and C. van Amerongen, Philips Res. Rept. 8, 452 (1953).

[2] I. R. Weingarten, Annual Report of the Conference on Electrical Insulation, 1955, Page 53. (More complete details on the low frequency bridge were obtained through private communication.)

[3] L. Heroux, J. App. Phys. 29, 1639 (1958).

[4] R. H. Cole and P. M. Gross, Jr., Rev. Sci. Inst. 20, 252 (1949).

[5] T. Nakajima, and D. Kondo, Bull. Electrotech. Lab. (Tokyo) 20, 641 (1956).

[6] T. Nakajima, and S. Saito, J. Poly. Sci. 31, 423 (1958).

[7] A. H. Scott, D. J. Scheiber, A. J. Curtis, J. I. Lauritzen, and J. D. Hoffman, Dielectric properties of semicrystalline polychlorotrifluoroethylene (Manuscript in preparation).

[8] A. H. Scott, J. Research NBS 50, 147 (1953).

[9] G. W. O. Howe, Wireless Engr. 12, 291 (1935).

[10] B. V. Hamon, Proc. Inst. Elec. Eng. 99, pt. IV (Monograph No. 27,1952$)$.

(Paper 65C1-52) 